\title{
Toward wearable supernumerary robotic fingers to compensate missing grasping abilities in hemiparetic upper limb
}

This is the peer reviewed version of the following article:

Original:

Hussain, I., Spagnoletti, G., Salvietti, G., Prattichizzo, D. (2017). Toward wearable supernumerary robotic fingers to compensate missing grasping abilities in hemiparetic upper limb. THE INTERNATIONAL JOURNAL OF ROBOTICS RESEARCH, 36(13-14), 1414-1436 [10.1177/0278364917712433].

Availability:

This version is availablehttp://hdl.handle.net/11365/1012982

since 2018-01-10T15:29:25Z

Published:

DOI:10.1177/0278364917712433

Terms of use:

Open Access

The terms and conditions for the reuse of this version of the manuscript are specified in the publishing policy. Works made available under a Creative Commons license can be used according to the terms and conditions of said license.

For all terms of use and more information see the publisher's website.

(Article begins on next page) 


\title{
Towards wearable supernumerary robotic fingers to compensate the missing grasping abilities in hemiparetic upper limb
}

Journal Tille

$\mathrm{XX}(\mathrm{X}): 1-23$

(C) The Author(s) 2016

Reprints and pormission:

sagepub.co.uk/journalsPermissions.nav

DOI: $10.1177 /$ ToBeAssigned

www.sagepub.com/

BSAGE

\author{
Irfan Hussain ${ }^{1}$, Giovanni Spagnoletti ${ }^{1}$, Gionata Salvietti ${ }^{1,2}$ and Domenico Prattichizzo ${ }^{1,2}$
}

\begin{abstract}
This paper presents design, analysis, fabrication, experimental characterization and evaluation of two prototypes of robotic extra fingers that can be used as grasp compensatory devices for hemiparetic upper limb. The devices are the results of experimental sessions with chronic stroke patients and consultations with clinical experts. Both devices share a common principle of work which consists in opposing to the paretic hand/wrist so to restrain the motion of an object. They can be used by chronic stroke patients to compensate for grasping in several Activities of Daily Living (ADL) with a particular focus on bimanual tasks. The robotic extra fingers are designed to be extremely portable and wearable. They can be wrapped as bracelets when not being used, to further reduce the encumbrance. Both devices are intrinsicallycompliant and driven by a single actuator through a tendon system. The motion of the robotic devices can be controlled using an Electromyography (EMG) based interface embedded in a cap. The interface allows the user to control the device motion by contracting the frontalis muscle. The performance characteristics of the devices have been measured through experimental set up and the shape adaptability has been confirmed by grasping various objects with different shapes. We tested the devices through qualitative experiments based on ADL involving five chronic stroke patients. The prototypes successfully enabled the patients to complete various bi-manual tasks. Results show that the proposed robotic devices improve the autonomy of patients in ADL and allow them to complete tasks which were previously impossible to perform.
\end{abstract}

\section{Keywords}

Wearable Robotics, Assistive Devices, Robotic Extra-Limbs, Supernumerary Fingers

\section{Introduction}

Robotic applications have rapidly grown from classical industrial applications with repetitive tasks to applications with close human-robot interaction. In particular, assistive robotics has gained an increasing attention in the last decades, see Van der Loos and Reinkensmeyer (2008). Assisting robotic devices can have a great impact on the adaptation of healthcare services to the needs of an increasingly dependent population (aging, degenerative diseases, etc.). Technological advances and the emergence of novel adapted technologies such as wearable technologies with considerable reduction in size, cost and energy consumption are becoming a popular solution to provide assistive services to humans. This capable technology is expected to work closely, interact and collaborate with people in an intelligent environment. While initially conceived for human motion augmentation purposes, wearable powered robots have been gradually proposed as a technological aid for motion rehabilitation and assistance, and functional substitution in patients suffering from motor disorders, see Pons et al. (2008).

In this work, we focus on a novel wearable assistive technology for chronic stroke patients. Stroke is one of the leading causes of a long term impairment. On average, every 10 seconds, someone in the United States has a stroke Go et al. (2014). Impairment of the hand

\footnotetext{
${ }^{1}$ Università degli Studi Siena, Department of Information Engineering, Via Roma 56, Siena - Italy

${ }^{2}$ Istituto Italiano di Tecnologia, Via Morego 30, Genoa - Italy

Corresponding author:

Irfan Hussain, Università degli Studi Siena, Department of Information Engineering, Via Roma 56, Siena - Italy

Email: irran.hussain@unisi.il
} 


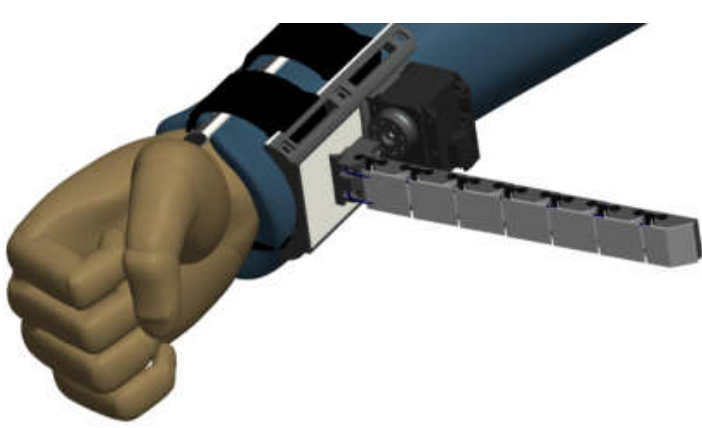

(a) The soft sixth finger concept.

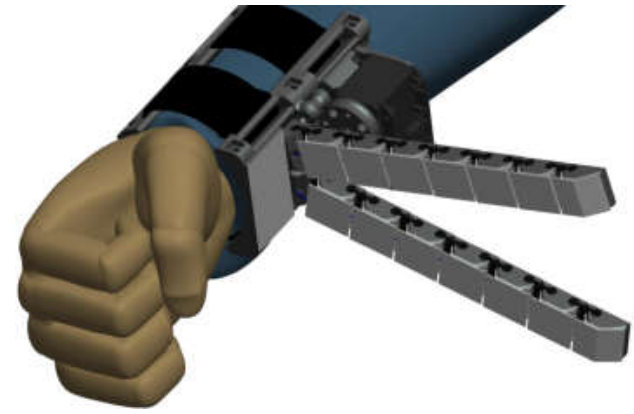

(b) The double soft sixth finger concept.

Figure 1. The proposed wearable robotic compensatory devices for hemiparetic upper limb. On the left, a single extra finger is proposed to compensate for missing hand grasping function. On the right, a double finger is proposed to increase the grasp stability and the payload.

grasping function is one of the common deficits after a stroke: approximately $60 \%$ of stroke survivors suffer from some form of sensorimotor impairment associated with their hand Nowak (2008). Different motor impairments can affect the hand both at motor execution and motor planning/learning level. Deficits in motor execution include weakness of wrist/finger extensors, increased wrist/finger flexors tone and spasticity, co-contraction, impaired finger independence, poor coordination between grip and load forces, inefficient scaling of grip force and peak aperture, and delayed preparation, initiation, and termination of object grip Raghavan et al. (2006); Balasubramanian et al. (2010). Recovering hand functions is of primary importance during the rehabilitation phase. Many wearable devices have been proposed in the last decade, especially for hand rehabilitation and function recovery. A revicw of robotassisted approaches to motor neurorchabilitation can be found in Lum et al. (2012). However, most of the devices are designed to increase the functional recovery in the first months of the rehabilitation therapy, when biological restoration and reorganization of the central nervous system can take place. However, only $5 \%$ to $20 \%$ of patients show a complete recovery of upper limb six months after the stroke Nakayama et al. (1994). When in the paretic upper limb the motor deficit is stabilized, the rehabilitation mainly consists in ergotherapy, with the primarily focus of teaching compensatory strategies that may often take advantage of dedicated aids. These strategies may sometimes result neither ergonomic nor ecological Davis and Burton (1991), even increasing the pathological motor patterns, by usually worsening tonic flexion at the forearm of the paretic limb Michaelsen et al. (2004). Various compensatory aids are commercially available in order to support patients in activities of daily living (ADI) Gillen (2015). The main target of the dedicated tools is to let typical bimanual tasks be executed using only the unaffected hand increasing the disparity between the two upper limbs. Moreover, these tools can be difficult to carry outside structured environments, limiting their use to rehabilitation facilities or to patient's house.

A possible solution is that of using a wearable compensatory robotic device that can work together with the paretic upper limb to compensate the missing abilities of the impaired hand. These devices should, for example, recover the ability to grasp and stabilize objects, while keep motivating the patients to use residual mobility of their paretic upper limb. Moreover, a wearable robotic device could easily be carried by patients even outdoors. Finally, a single robotic device could replace many commercially available tools to perform $\Lambda \mathrm{DL}$, sinec these tools are gencrally designed to perform a single task.

Existing compensatory robotic devices like prostheses, cannot be used for this purpose since the hand of the patient, although frequently with limited mobility, is still present. Early results on the replacement of the impaired hand with robotic devices are reported in Aszmann et al. (2015). However, this potential solution could be much less effective in chronic stroke patients where the whole arm often presents a limited residual mobility. Exoskeletons are another kind of assistive devices, where an external mechanism with joints and links is coupled with the corresponding joints of the human limbs. Heo et al. (2012) presented a comprehensive review of hand exoskeleton technologies for rehabilitation and assistive engineering. Rigid exoskeletons do not accommodate variations in patient skeletal structure or joint misalignment and can produce compression forces on the soft tissue and joints during longterm use Pons et al. (20)4). Moreover, most of the proposed exoskeletons are cumbersome limiting the wearability and portability of the device. 
Besides exoskeletons and prostheses and their working principles, it is interesting to study other robotic solutions which can compensate the missing grasping function. The aim is to come up with a robotic device that can work together with the paretic upper limb instead of replacing it and without causing any unnatural forces. In robotics, onc of the simplest structure that allows to grasp is the gripper. Industrial grippers usually have two fingers and only one degree of freedom. Further simplifying, one finger can be seen as a fixed palm and the other one as an active finger able to restrain the motion of an object. If we consider the paretic upper limb of the patient as a potential fixed palm, what is really missed is an active finger able to perform the grasp. In this view, robotic extra fingers can represent the minimal complexity solution that also guarantee extreme wearability and that do not require to be coupled with human impaired limbs in order to compensate for missing capabilities. Such devices could enhance manipulation capability without relying on the user's skeletal structure for support, making anatomical variation and motion restriction a lesser issue.

Recently, the research groups at the Massachusetts Institute of Technology and at the University of Siena independently developed ideas and prototypes of robotic extra fingers. Wu and Asada (2014) presented the design of two supemumerary robotic fingers. A method for controlling extra robotic fingers in coordination with human fingers to grasp diverse objects has been further developed in Wu and Asada (2016). Although two independent extra fingers showed great potentialities in augmenting human hand functions in healthy subjects, size and proposed control strategies limit their possible application as assistive device. Toward the direction of active assistive devices, we have started investigating in Prattichizzo ct al. (2014a), Prattichizzo et al. (2014b) a wearable robotic extra finger to be used in cooperation with the parctic limb to recover the capability of grasping objects. In Hussain ct al. (2015b) we have reported some preliminary results on the use of a modular robotic structure as an active compensatory tool for chronic stroke patients. In particular, the extra finger prototype was worn on the paretic forearm by means of an elastic band, and it was coupled with a vibrotactile ring interface worn on the healthy hand. The robotic finger and the paretic hand acted like the two parts of a gripper working together to hold an object. The user was able to control the flexion/extension of the robotic finger through a switch placed on the ring, while being provided with vibrotactile feedback about the forces exerted by the robotic finger on the environment. In Salvietti et al. (2016) we introduced an EMG interface to control the finger flexion/extension as well as a compliance control to let the finger adapt to the shape of the grasped object. Finally, in Hussain et al. (2016) we proposed a preliminary version of an underactuated compliant extra finger. The proposed robotic devices were used by patients to perform clinical assessment tests, but the systems had limitations in performing common $\mathrm{ADL}$. Thosc limitations were associated to the performance characteristics of the robotic devices and to the limited capability of a single finger to perform particular tasks, e.g., pouring water from a bottle. The device limitations were mainly due to low actuator torque, low friction at contact areas and weak coupling of the device at the forearm.

In this work, we present two novel prototypes of wearable grasp compensatory devices for hemiparetic upper limb: the soft sixth finger and the double soft sixth finger, sec Figure 1.

The former is an improved version of the device presented in IIussain et al. (2016). The new design shares some common features with the previous device in terms of wearability, robustness and ability to adapt to different object shapes. However, the upgraded version has a new actuator with increased torque, silicone skin to increase friction, double tendon actuation to improve torsional rigidity and a new support base design to provide stable grip at the forcarm. The double soft sixth finger device has been designed to improve grasp stability in more payload demanding tasks. We doubled the flexible structure of the device to obtain two fingers. The two fingers are attached to a base that can be worn at the user's forearm. The device is actuated by a single motor and the two fingers design improves the grasp stability and robustness. An improved version of the eCap, an EMG interface embedded in a cap proposed in its preliminary version in Hussain et al. (2016), is also presented in this paper.

We evaluated the performances of the assistive system through an experimental setup. The results showed major improvements in the performance characteristics of the devices, in particular, grasp stability, fingertip force and maximum payload. Such improvements led to successful experiments with the patients in performing various ADL tasks. We tested the devices with five patients selected by the clinical team based on the criteria explained in Section 4. The experiments focused on bi-manual activities related to the kitchen and on the use of mechanical tools. During all the tasks patients used the robotic finger and the paretic limb to hold the object while the unaffected hand was used to perform manipulation on it. One of the main contributions of this work is the evaluation of the effectiveness of wearable extra robotic fingers as compensatory tools for chronic stroke patients in ADI, where bi-manual tasks are involved. The 


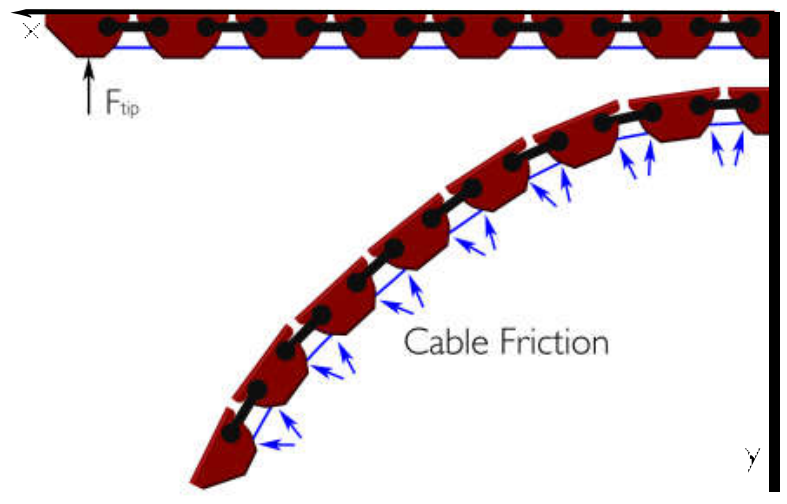

Figure 2. Underactuated cable driven flexible finger. The finger has modular structure. Each module is composed of soft and stiff parts.

rest of the paper is organized as follows. In Section 2, we present the mathematical model of the robotic finger used for determining device specifications, design, fabrication and experimental characterization of the proposed robotic devices based on flexible structure. The control interface is detailed in Section 3, while the experiments with chronic stroke patients are presented in Section 4. Results and discussion are explained in Section 5. Finally, conclusion and future work are outlined in Section 6.

\section{The soft sixth finger and the double soft sixth finger}

The wearable assistive robotic devices used for clinical applications must meet specific human factors and performance criteria. The general guidelines that can be found in the literature include: durability, encrgy cfficiency, low-encumbrance, case of use, error tolerance and configurability Meng and Lec (2006). The specific performance critcria and human ergonomics strongly depend on the actual patient conditions and necds Stanger et al. (1994); Miguclez et al. (2004). In order to improve the wearability and the acceptability of the devices for the users, the design must meet a number of conditions related to ergonomics and functionality Pons (2010). Several experiments with the patients conducted in cooperation with a rehabilitation team reported in Hussain et al. (2015b, 2016); Salvietti et al. (2016) led us to outline the main functional requirements and human factors reported in Table 1. We considered these ergonomics and functional requirements in the design and development of the proposed robotic devices. Table 8 summarizes how we met these requirements in our devices while the detailed design and development methodologies are explained in corresponding sections. The compensatory devices have been designed to be wearable, robust and capable to adapt to different object shapes. In general, the robustness plays a twofold role. First, it enables the robotic devices to reliably grasp objects in the presence of large sensory uncertainty. Second, it enables the devices to withstand large impact and other forces duc to the unintended contact with the environment. The robustness and soft interaction are mainly achieved by either regulating the compliance of robotic joints Vanderborght et al. (2013) or tuning the intrinsic softness, acting on the passive characteristics of the robot bodyware Dollar and Howe (2011); Laschi and Cianchetti (2014); Ma et al. (2015). The former approach is based on complex and often bulky variable impedance actuators. Our devices are inspired by the latter approach in order to be simple, light in weight and compact in size. The proposed devices are based on the principle of an underactuated cable driven flexible and modular structure as shown in Figure 2. The passive compliance in the joints guarantees the robustness and safety during the interaction with the environment. The devices can endure collisions with hard objects and even strikes from a hammer without breaking into pieces.

In addition to this, the actuation system of the proposed devices resembles that of underactuated robotic hands Birglen et al. (2008). Underactuated hands have desirable adaptability to shapes, and can be effectively implemented using relatively simple differential and elastic clements. The transmission solutions allow motion of other joints to continue after contact occurs on a coupled link, allowing the hands to passively adapt to the object shape Dollar and Howe (2010). Passive adaptability allows to drive the device with a reduced number of control parametcrs. The built-in compliant nature of the extra-fingers and underactuation increase their ability to grasp different objects. They can adapt their shape to that of the grasped object. Shape adaptation increases the grasp performance by compensating the uncertainties in sensing, actuation and helps in stabilizing the grasp Eppner and Brock (2013). The robustness and intrinsic compliance is realized through the cable driven flexible structure of the robotic fingers.

A mathematical model, presented in Section 2.1, has been used to study the kinematics of such cable driven flexible finger and to simulate its bending profile. The simulation results helped in minimizing the manufacturing iterations, in particular adjusting the stiffness in each joint to obtain a desired finger closing trajectory and length of finger to cover a certain workspace. In Section 2.2 the detail of design, development and performance evaluation of the soft sixth finger are explained. Section 2.3 describes the design, development and preliminary evaluation of the double soft 
Table 1. Ergonomics and functional requirements

\begin{tabular}{|c|c|c|}
\hline Category & Requirement & Literature/Experimental Observations \\
\hline \multicolumn{3}{|c|}{ Ergonomics } \\
\hline & Wearability & $\begin{array}{l}\text { To be wearable the device should be of low- } \\
\text { encumbrance Casson et al. (2010). }\end{array}$ \\
\hline & Portability & $\begin{array}{l}\text { To be used also outside the laboratory and structured } \\
\text { cnvironment in outdoor applications Webb ct al. (2012). }\end{array}$ \\
\hline & Weight & $\begin{array}{l}\text { To minimize the weight at the forcarm. The arm } \\
\text { impairment causes less mobility and capability to lift } \\
\text { and carry loads Hussain et al. (2016). Lightweight }(< \\
400 \mathrm{~g} \text { ) Pons et al. (2004). }\end{array}$ \\
\hline & Ease of use & $\begin{array}{l}\text { To provide freedom to patients in wearing and using the } \\
\text { device without any assistance Stanger et al. (1994). }\end{array}$ \\
\hline \multicolumn{3}{|l|}{ Functional } \\
\hline & Robustness & $\begin{array}{l}\text { To resist to unwanted collisions with the environ- } \\
\text { ment Odhner et al. (2014). }\end{array}$ \\
\hline & $\begin{array}{l}\text { Fatigue avoidance and } \\
\text { safety }\end{array}$ & $\begin{array}{l}\text { To avoid un-natural forces on human muscles during } \\
\text { the use of device which can cause fatigue and } \\
\text { frustration Pons et al. ( } 2008) \text {, the human-robot safe } \\
\text { interaction must be ensured Bicchi ct al. (2008). }\end{array}$ \\
\hline & $\begin{array}{l}\text { Device adaptability to } \\
\text { patient's conditions }\end{array}$ & $\begin{array}{l}\text { The device positioning according to patient's conditions } \\
\text { and residual mobility of the arm/hand Salvietti et al. } \\
(2016) \text {. }\end{array}$ \\
\hline & $\begin{array}{l}\text { Control interface adaptabil- } \\
\text { ity to paticnt's conditions }\end{array}$ & $\begin{array}{l}\text { The bio-signal control interface must be adaptive to } \\
\text { patient dependent nature of signal variations and given } \\
\text { detection conditions Konrad (2005). }\end{array}$ \\
\hline & $\begin{array}{l}\text { Device coupling with human } \\
\text { arm }\end{array}$ & $\begin{array}{l}\text { To firmly couple the device with human forearm, in order } \\
\text { to realize a stable grip even in the presence of heavy } \\
\text { loads T Walley Williams III et al. (2011). }\end{array}$ \\
\hline & Object shape adaptability & $\begin{array}{l}\text { To adapt to objects with different shapes and sizes Dollar } \\
\text { and Howe }(2010) \text {. }\end{array}$ \\
\hline & Grasp stability & $\begin{array}{l}\text { To realize a stable grasp during holding, lifting and } \\
\text { pouring tasks, involving objects of various sizes and } \\
\text { weights. }\end{array}$ \\
\hline & Mechanical power & $\begin{array}{l}\text { To generate the contact forces }(5 N) \text { required for } \\
\text { successfully grasping objects of daily living Belter and } \\
\text { Segil (2013). }\end{array}$ \\
\hline & Configurability & $\begin{array}{l}\text { To casily adjust according to paticnt specific necds, c.g., } \\
\text { the desired control interface, the length of the device, } \\
\text { casy asscmbly of parts, ctc. Stanger et al. (1994) }\end{array}$ \\
\hline & Functional versatility & $\begin{array}{l}\text { To accommodate a wide varicty of objects and tasks } \\
\text { associated with ADL Graf (2008). }\end{array}$ \\
\hline & Energy efficiency & $\begin{array}{l}\text { To avoid expensive, unsafe and heavy energy } \\
\text { sources Webb el al. (2012). }\end{array}$ \\
\hline & Error tolerance & $\begin{array}{l}\text { To provide robustness to positioning and sensing errors, } \\
\text { and having mechanical design features suited to grasp } \\
\text { stability including: high friction, natural distributed } \\
\text { compliance and underactuation Dollar and Howe (2010). }\end{array}$ \\
\hline & $\begin{array}{l}\text { Simple and intuitive inter- } \\
\text { faces }\end{array}$ & $\begin{array}{l}\text { To realize simple and user friendly control interface Bel- } \\
\text { ter and Segil (2013). }\end{array}$ \\
\hline
\end{tabular}

sixth finger. Wearability and device position at forearm according to patient's condition are detailed in Section 2.4. 


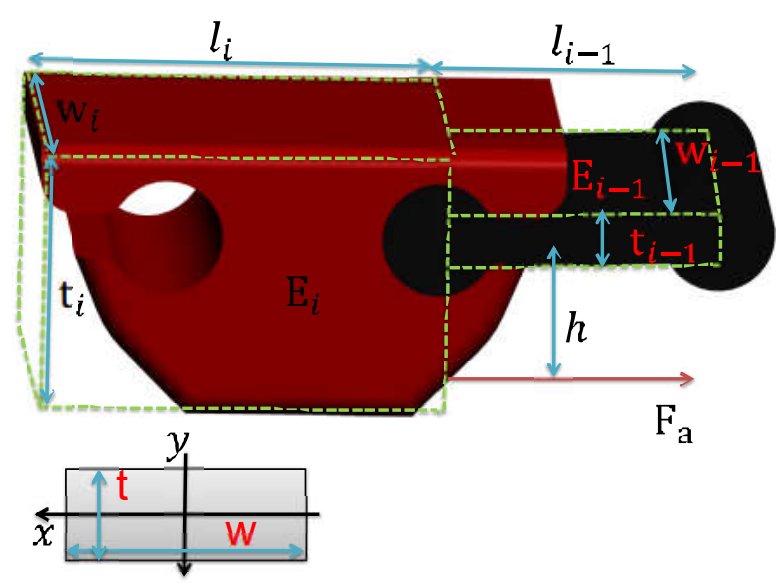

Figure 3. Module geometric and material parameters are shown. The module consists of flexible part $(i-1)$ and stiff part (i).

\subsection{Static analytical model and analysis}

A mathematical model of a cable driven flexible finger is developed to minimize the number of manufacturing iterations and to study the kinematics of flexure-based multijointed robotic finger. In particular, the model is analyzed:

1. To determine the fingertip deflection in response to actuator's force.

2. To observe joints deflection variation by changing the density of flexible part.

3. To estimate the minimum size of self enveloping graspable object with selected length of the finger and deflection (stiffness) in each joint.

Unlike simple revolute joint-based serial kinematic chains, the flexural nature of flexible fingers necds a model of flexural mechanics coupled with a kinematic model to study the finger behavior. $\Lambda$ model for a flexible robotic gripper is proposed in Gafford et al. (2014), where the trajectory of the finger is regulated by choosing the different lengths of each link. In this work, we adapt that model in order to act on the stiffness of flexible joints to regulate the trajectory of the proposed flexible modular finger.

In order to derive a static analytical model, let us consider a single module as that shown in Figure 3, composed of a flexible part (subscript $i-1$ ) and a stiff part (subscript $i$ ). The actuator applies force $\left(F_{a}\right)$ through the tendon which produces the moment $\left(F_{a} h\right)$ about the llexible joint. The model assumes that the deformation behavior is dominated by the actuator applied moment, while the fingertip and gravity contribution and reaction forces in the $\mathrm{x}$-direction are negligible. Let us consider a point $p_{i}\left(\delta_{i}, l_{i}\right)$ that describes the position and orientation of the module $i$, where $\delta_{i}$ denotes the local deflection between the part $i$ and $i-1$ and $l_{i}$ is the length of the part according to the notation introduced in Timoshenko and Gere (1972). The local deflection in response to an applied force can be computed as

$$
\delta_{i}=\frac{F_{a} h l_{i}^{2}}{2 E_{i} I_{i}}
$$

where $E_{i}$ is the Young's modulus of clasticity, $I_{i}$ is the sccond moment of arca, $l_{i}$ is the length of the modulc part and $h$ is the distance between tendon pulling force vector and neutral axis. Let $\theta_{i}$ denotes the angle between the parts $i$ and $i-1$

$$
\theta_{i}-\frac{-F_{a} h l_{i}}{E_{i} I_{i}}
$$

For the sake of simplicity, we neglect the deflection of the joint in the lateral and torsional direction. This simplification was validated through finite element method based simulations and stress/deformation analysis presented in Hussain et al. (2017a). We observed that, for our designed geometry of the flexible joint, the lateral and torsional stiffness is higher than the bending one.

The rotational stiffness of the joint can be then evaluated as

$$
k_{i}-\frac{E_{i} I_{i}}{l_{i}}
$$

Tendon pulling force $\left(F_{\alpha}\right)$ can be computed by the torque $\left(T_{m}\right)$ exerted by the motor and pulley radius $\left(r_{p}\right)$

$$
F_{a}-\frac{T_{m}}{r_{p}}
$$

In order to achieve a certain closing trajectory of the flexible finger, we need to have a different deflection in each joint, under the same applicd force. In Hussain et al. (2017a), we proposed a detailed mathematical framework to design and develop the stiffness of flexible joints necessary to generate the desired trajectory of the supernumerary robotic fingertip inspired by first syncrgy of human hand. Here, we recall the main part of the model which focuses on the hardware realization of soft joints with given stiffness. Referring to Equation 1, different deflections and joint angles under the same applied force can be achieved by changing the geometric or material parameters of the modules. Since we want a modular structure, the geometric parameters are the same for each module. On the other hand, we can tune the material parameters in order to achieve variable joint deflection of robotic finger. In particular, by keeping in view the intended $3 \mathrm{D}$ printing fabrication method, we can transform the above mentioned equations in terms of a density parameter. Thus, flexible parts can be printed with different percentages of infill density to get different 
Table 2. Material and geometric parameters of module's flexible and stiff parts. Where, $h=6 \mathrm{~mm}$, constant for each module.

\begin{tabular}{|c|c|c|}
\hline $\begin{array}{l}\text { Material } \\
\text { Parameters }\end{array}$ & Flexible Part & Stiff Part \\
\hline Material type & $\begin{array}{l}\text { Thermoplastic } \\
\text { polyurethanc } \\
\text { (TPU) }\end{array}$ & $\begin{array}{l}\text { Acrylonitrile } \\
\text { Butadicne } \\
\text { Styrene } \\
(A B S)\end{array}$ \\
\hline $\begin{array}{l}\text { Modulus of elasticity } \\
(E)\end{array}$ & $15.2 M P a$ & $10 M P a$ \\
\hline Shorc Hardness & $85 \Lambda$ & $70 D$ \\
\hline \multicolumn{3}{|l|}{$\begin{array}{l}\text { Geometric Parame- } \\
\text { ters }\end{array}$} \\
\hline$w_{i}$ & $21 \mathrm{~mm}$ & $21 \mathrm{~mm}$ \\
\hline$l_{i}$ & $13 \mathrm{~mm}$ & $20 \mathrm{~mm}$ \\
\hline$t_{i}$ & $2.5 \mathrm{~mm}$ & $12 \mathrm{~mm}$ \\
\hline \multicolumn{3}{|l|}{$\begin{array}{l}\text { 3D printing infill } \\
\text { density percentage }\end{array}$} \\
\hline 1 (Base module) & $50 \%$ & $100 \%$ \\
\hline 2 & $40 \%$ & $100 \%$ \\
\hline 3 & $30 \%$ & $100 \%$ \\
\hline 4 & $20 \%$ & $100 \%$ \\
\hline 5 & $30 \%$ & $100 \%$ \\
\hline 6 & $10 \%$ & $100 \%$ \\
\hline 7 (Fingertip) & $50 \%$ & $100 \%$ \\
\hline
\end{tabular}

deflection while maintaining the same geometric shape of the parts.

The density $\left(\rho_{i}\right)$ of a part is its mass $\left(m_{i}\right)$ per unit volume $\left(V_{i}\right)$

$$
\rho_{i}=\frac{m_{i}}{V_{i}}=\frac{m_{i}}{l_{i} w_{i} t_{i}} .
$$

Thus, rewriting Equation 1 and Equation 2 we get

$$
\begin{aligned}
\delta_{i} & =\frac{-F_{a} h m_{i}^{2}}{2 E_{i} I_{i} \rho_{i}^{2} w_{i}^{2} t_{i}^{2}}, \\
\theta_{i} & =\frac{-F_{a} h m_{i}}{E_{i} I_{i} \rho_{i} w_{i} l_{i}} .
\end{aligned}
$$

Assuming both parts of the module as filled rectangular shape whose centroid is located at the origin, the second moment of area with respect to $\mathrm{x}$-axis can be approximated as

$$
I_{i}-\frac{w_{i} l_{i}^{3}}{12}
$$

The mathematical model can be extended to any number of modules. We selected seven modules in order to achieve the length of the finger equal to average size of human hand Schwarz (1955), approximately. We simulated the model of the complete flexible finger adapting the model available on the open source platform Soft Robotics Toolkit Ilolland et a1. (2014). In particular, we added the possibility to change the stiffness of the soft joints by acting on the the percentage of infill density. The overall deflection at the fingertip can be determined by propagating the internal moments generated by the tendon tension back to the base modulc. We defined the global frame of reference at the base module and transformed the local deflection into global frame using homogeneous transformation matrices given in Appendix A. We simulated the analytical model with different density percentage for each joint to set the trajectory of the finger for maximum enveloping grasp. The final density percentage for each module is listed in Table 2. We 3D printed the flexible parts with resultant percentage of infill density to achieve different deflection in joints under same applied tendon force. We estimated the minimum size of a self enveloping graspable object as $2.3 \mathrm{~cm}$, with a selected length of the finger of $18 \mathrm{~cm}$.

The model proposed above can be further exploited to relate the fingertip force to the actuator force. The force applied by the actuator through the tendon produces a moment about the flexible part of the finger. The resultant behavior can be approximated by a simplified cantilever beam model. The two forces acting on the model are the actuator applied force and the resultant reaction force which is equal to the fingertip applied force $F_{t i p}$. We can study the deflection effect generated by the two forces separately by using the superposition principle. Let $\delta_{a}$ be the deflection due to the actuator applied force and $\delta_{r}$ be the deflection due to the reaction force

$$
\begin{aligned}
& \delta_{a}=\frac{F_{a} h l^{2}}{2 E I}, \\
& \delta_{r}=\frac{F_{l i p} l^{3}}{3 E I} .
\end{aligned}
$$

The sum of both deflections can be equated to zero and the resultant equation is solved for $F_{t i p}$

$$
F_{l i p}=\frac{3 F_{a} h}{2 l}
$$

Note that the terms $E$ and $I$ are cancelled out of the cquation and as a result we do not need to consider the interaction between the alternating stiff and flexible parts of the modules to obtain the overall load at the fingertip. The model can be extended to any number of modules, as

$$
F_{l i p}=\frac{3 F_{a} h}{2 \sum_{i-1}^{2 N} l_{i}} .
$$



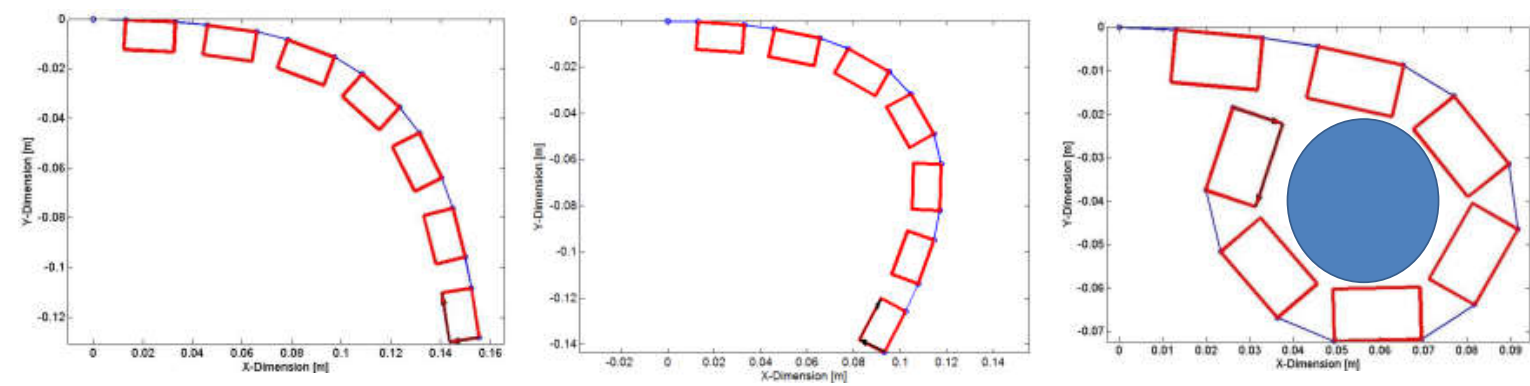

Figure 4. Simulation results of flexible finger trajectory: flexible part (blue) and stiff part ( $r e d)$. Different closing trajectories of finger are plotted in left, center and right plot. The trajectories variation are achieved by setting different density percentage of flexible parts. The trajectory selected for the soft sixth finger is reported in the plot on the right

Furthermorc, we can consider the parasitic capstan effects that take place betwecn the cablc and stiff parts as the robotic finger transforms to a curve shape as shown in Figure 2. As the modules lose the colinearity during their motion, the cable imparts a reaction force that resists further actuation. We can include the capstan effect by considering the angle between subsequent stiff parts of modules $\left(\theta_{i-1}-\theta_{i}\right)$. The tendon does not pass through the hexible parts, so we can only consider the parasitic capstan effects on stiff parts. Thus, the fingertip force can be modified as

$$
F_{\text {tip }}=\frac{3 F_{a} h}{2 \sum_{i=1}^{2 N} l_{i}} \prod_{i=2, \text { cven }}^{2 n-2} e^{\mu\left(\theta_{i}-\theta_{i}\right)}
$$

where $\mu$ is the friction coefficient.

Figure 4 shows the simulation results of flexible finger trajectories based on the presented mathematical model. We simulated the model with different density percentage for Mexible parts to observe the finger trajectory variation. The final selected density percentage of each flexible joint for the developed prototype is listed in Table 2. The selected trajectory is reported in the right side of Figure 4.

We experimentally validated our method through an experimental setup. We built the soft finger considering the different percentage of infill density for the soft joints reported in Table 2. We tracked the fingertip trajectory of the device by using a Vicon system (Vicon Capture Systems. UK) consisting of 8 cameras. We compared the mean of five recorded trajectory of the fingertip with the one computed through the mathematical model as reported in Fig. 5. The mean error between the simulated trajectory and the experimentally evaluated one was $0.023 \mathrm{~m}$ along $x$-axis and $0.021 \mathrm{~m}$ along $y$-axis, while the maximum error was $0.04 \mathrm{~m}$ and $0.027 \mathrm{~m}$ for $x$ and $y$ axes, respectively. This error is due to different factors including the 3D printed accuracy in density regulation, unmodelled friction between the tendon and the stiff part of the modules and small finger fluctuation

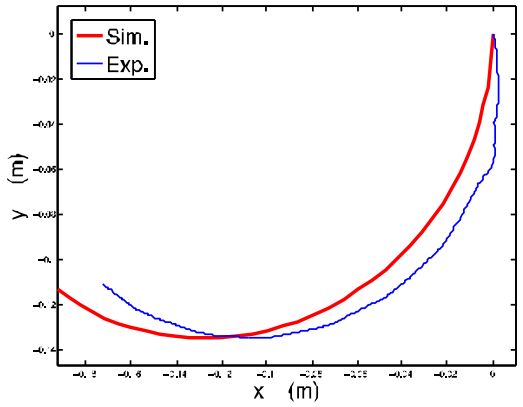

Figure 5. Trajectory of the fingertip of the soft sixth finger simulated $(r c d)$ and experimentally evaluated $(b l u c)$. Simulation are obtained using the model presented in Sec 2.1. Experimental data are obtained using an optical tracking system.

during the flexion motion. Ilowever, this error did not impact on the usability of the extra-finger as reported is Section 4.

\subsection{The Soft Sixth Finger}

2.2.1 Design and Manufacturing The soft sixth finger is composed of two main parts, a flexible finger and a support base as shown in Figure 6. The flexible finger is built with a modular structure. Each module consists of a rigid 3D printed link realized in ABS (Acrylonitrile Butadiene Styrene, ABSPlus, Stratasys, USA) and covered with a silicon skin and a $3 \mathrm{D}$ printed thermoplastic polyurethane part (Lulzbot, USA) that acts as the flexible joint. We selected polyurethane for flexible parts because the high elongation of this material allows for repeated movement and impact without wear or cracking proving also an excellent vibration reduction. Reasons for adding passive elements are manifold, including storing clastic energy, avoiding tendon slackness, passive compliance, the distribution of forces over a large contact area and ensuring the uniqueness of the position Catalano et al. (2014).

The modules are connected by sliding the thermoplastic polyurethane part in the ABS part. This method makes the assembling process easy without using any screws or passive 


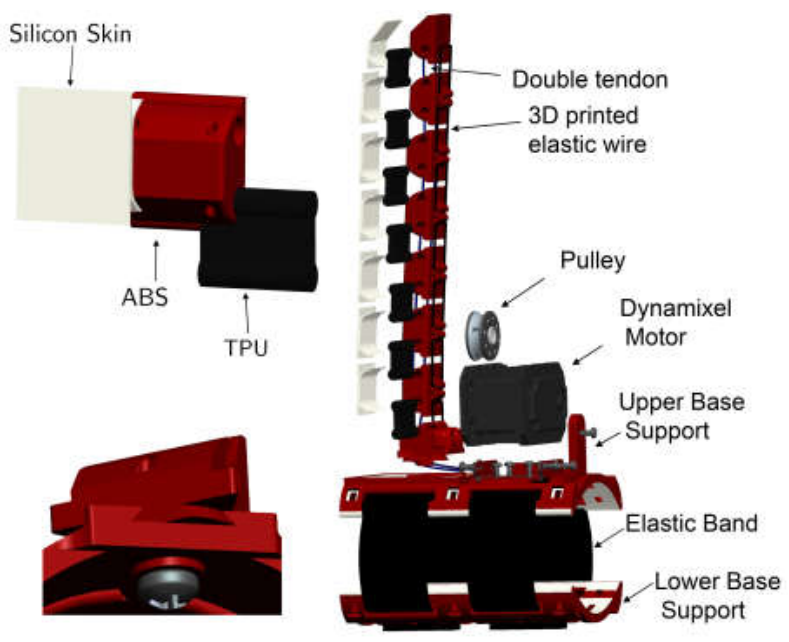

Figure 6. The CAD exploded view of unit module, complete soft sixth finger and Dovetail passive locking mechanism. Two holes in the module for double tendon, modular structure of the device, support base and actuator are shown.

elements to combine the modules. The length and closing trajectory of the device have been selected according to the procedure presented in Section 2.1. The device is designed and developed by combining two different manufacturing processes, i.e., 3D printing and moulding. The skeleton of the device is fabricated by rapid prototyping $3 \mathrm{D}$ printing while the silicone skin is realized by a moulding process. The moulding process shapes the raw material using a solid frame of a particular shape, called a pattern. We used 3D printed skeletons to hold the liquid silicon in desired shape until it turned solid. We realized closed-top moulds which are used for more complex part geometries. We poured the silicone mixture over the skeletons of the modules and support base and used other mold's parts to constrain the liquid silicone to achieve the desired geometry and shape of the skin. Metal tubes were inserted into the module holes so to avoid silicone filling the tendon holes. In Figure 7, the top row shows the exploded view of the parts used in the moulding process, the bottom row shows the assembled configuration of parts during the curing process of liquid silicon. The silicone used is Fast Rubber FR-18 which is bi-component and cures at room temperature. The mixing ratio of components are $100 \mathrm{~g}$ of resin per $5 \mathrm{~g}$ of hardncr. It has viscosity of $30 \mathrm{~Pa} \cdot \mathrm{s}$ and the final hardness is $17 \pm 2$ shore $\mathrm{A}$. The silicone skin on the rigid links is realized through casting process, aiming to increase the friction at the possible contact areas. The mold parts used in casting process are shown in Figure 7.

The support base of the device has been designed to assure a firm grip on the arm. The ergonomic design of the support base guarantees the stability of the device to withstand the
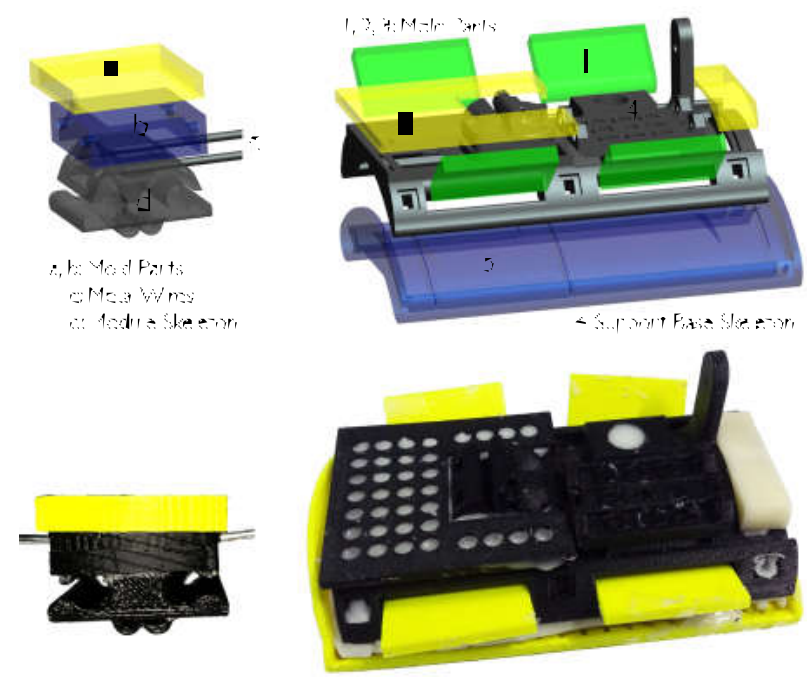

Figure 7. Moulding process to realize silicone skin. Mold parts, module skeleton and support base skeleton are 3D printed. Metal wires are inserted in the tendon wire holes to avoid silicon infiltration.

applied load. The support base consists of two parts coupled with velcro strips to facilitate wearing the device at the forearm and guarantee adaptation to different arm sizes. The upper part contains the actuator and base module of the robotic finger. Both parts are covered with a silicone skin to increase comfort and stability at the forearm. The skeleton of the support base is 3D printed by ABS material and the silicone skin is realized through moulding process. The support base and the flexible finger are coupled through Dovetail triangle locking mechanism, which is described in detail in Section 2.4. This mechanism is used to switch between working and rest position of the devices. The structure of the support base is symmetrical, feature that enables the robotic devices to be worn on both the left or the right arm of the patients without any modification in the device.

The device actuation is achieved by using a single actuator and two tendons running in parallel through the modules of the finger. The holes in the rigid links allow the passage of the cables (polyethylene dyneema fiber, Japan) which are used to realize the tendon driven actuation. The tendon wires run through the finger and are attached on one side to the fingertip and on the other to a pulley rigidly connected to the actuator shaft. When the motor is actuated, the tendon wires are wound on the pulley reducing the length of the wire and thus flexing the finger. As the motor is rotated in opposite direction, the extension of the finger is achieved thanks to elastic force stored in the flexible parts of the modules. The actuator used is the Dynamixel MX-28T (Robotis, South Korea). Principal details on the motor features are reported in Table 3, while for a complete 

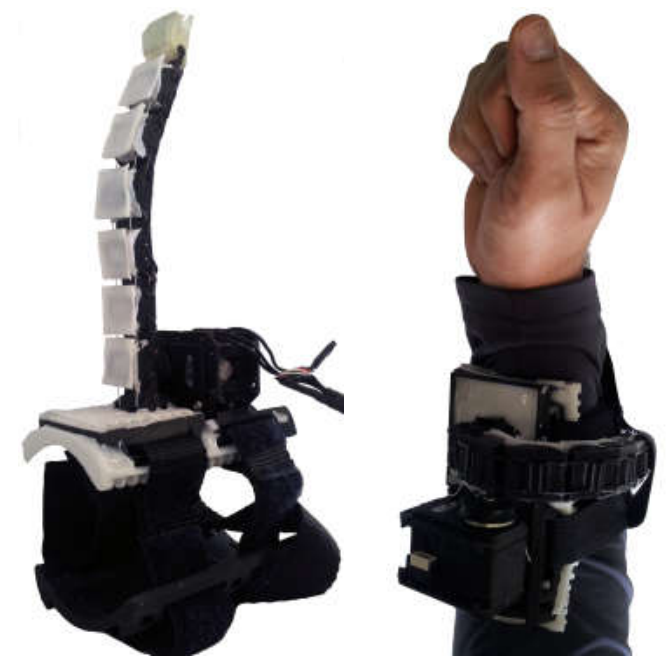

(a) Complete prototype of soft (b) The soft sixth finger as a sixth finger with silicone skin. bracelet during rest position.

Figure 8. The complete prototype of the soft sixth finger with silicone skin. The device can be worn on the arm using elastic band. It can be shaped as bracelet when being not used to reduce the encumbrance of the device.

description, the reader is referred to Robotis (2012). We use ArbotiX-M Robocontroller ArbotiX (2012) to drive the Dynamixcl motor. This control solution for Dynamixel motors incorporates an AVR microcontroller, a socket for a Xbee wireless radio and the motor driver.

The final prototype of the robotic device is reported in Figure 8 , while the technical details are summarized in Table 3.

2.2.2 Performance Characterization The device performances were evaluated through a subset of the tests proposed in Falco et al. (2015). In particular, we measured the maximum fingertip force, the maximum payload and maximum horizontal grasp resistive force.

The maximum fingertip force of the device was recorded while fixing its support base on a table with the finger perpendicular to the table surface. The initial configuration of the finger was fully extended and it was commanded to close at the maximum torque. The hook of a dynamometer (Vernier, USA) was rigidly coupled with the fingertip of the device so that force could be measured in vertical direction as shown in Figure 9-(a). The constant applied force value at fingertip is presented in Table 3. The maximum horizontal grasp resistive force was measured by grasping an object (diameter $=65 \mathrm{~mm}$, weight $=400 \mathrm{~g}$ ) with the robotic device and the arm. The object was slowly pulled horizontally by using the hook of the dynamometer, see Figure 9-c. It was measured that the grasp remained stable till $13 \mathrm{~N}$.
To check the maximum payload, an operator wore the grasp compensatory robotic device. The operator's arm was stabilized on a table while grasping a cylindrical object (diameter $=65 \mathrm{~mm}$, weight= $=400 \mathrm{~g}$ ) with the aid of the robotic tool at its maximum actuator's torque. The grasped object was slowly pushed down using the dynamometer's bumper as shown in Figure 9-(d). The maximum pushing force was recorded when the object started to slip. The maximum payload of the device (sec, Table 3) is the sum of grasped object's load and the one due to the pushing force. Figure 9(b) and Figure 9-(c) show the minimum size graspable objects. The diameter of the smallest graspable object is reported in Table 3.

In order to prove the grasping ability of the device and its shape adaptability to different objects, we used a subset of the objects included in the YCB grasping toolkit Çalli et al. (2015). This toolkit is intended to be used to facilitate bench-marking in prosthetic design, rehabilitation research and robotic manipulation. The objects in the set are designed to cover a wide range of aspects of the manipulation problem. It includes objects of daily life with different shapes, sizes, textures, weight and rigidity. We tested the device with different objects to evaluate how the robotic finger can adapt

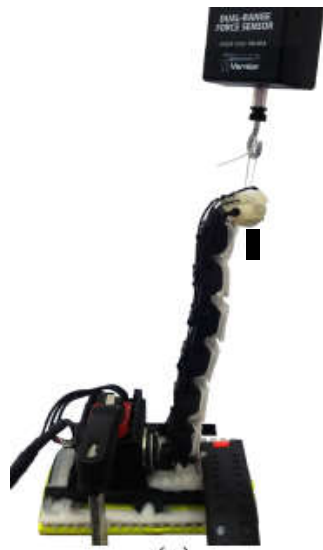

(a)

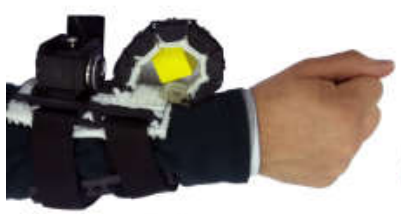

(b)

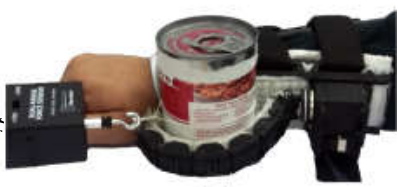

(c)

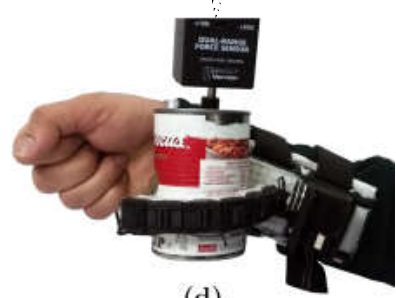

(d)

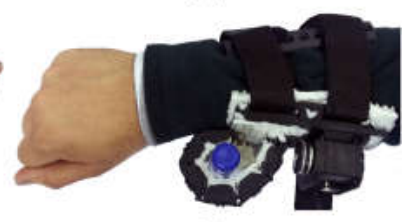

(e)
Figure 9. Experimental procedure to measure the performance characteristics of the soft sixth finger. The Figure (a), (c), (d) show the experimental setup for maximum fingertip force, horizontal grasp resistive force and maximum payload, respectively. The arrow in figure show the direction of applied force. Figure (b) and (e) show the minimum size graspable objects. 
Table 3. Technical details of the Soft Sixth Finger (SSF) and the Double Soft Sixth Finger (DSSF)

\begin{tabular}{|c|c|}
\hline Dimensions & \\
\hline Module & $20 \times 31 \times 12 \mathrm{~mm}^{3}$ \\
\hline Total length of finger (on arm) & $180 \mathrm{~mm}$ \\
\hline Support base & $110 \times 63 \times 3.5 \mathrm{~mm}^{3}$ \\
\hline Actuator control unit box & $71 \times 71 \times 15 \mathrm{~mm}^{3}$ \\
\hline \multicolumn{2}{|l|}{ Weights } \\
\hline Module & $4 \mathrm{~g}$ \\
\hline Actuator control unit box & $146 \mathrm{~g}$ \\
\hline \multicolumn{2}{|l|}{ Actuator } \\
\hline Max. torque & $3.1 \mathrm{Nm} 12 \mathrm{~V}$ \\
\hline Pulley radius & $8 \mathrm{~mm}$ \\
\hline Max. current & $1.4 \mathrm{~A} @ 12 \mathrm{~V}$ \\
\hline Continuous operating time & $3.5 \mathrm{~h} @$ stall torque \\
\hline Max. operating angles & $300 \mathrm{deg}$, endless turn \\
\hline Max. non-loaded velocity & $684 \mathrm{deg} / \mathrm{sec}$ \\
\hline \multicolumn{2}{|l|}{ The SSF performances } \\
\hline Max. Force at fingertip & $10 \mathrm{~N}$ \\
\hline Max. payload & $2.4 \mathrm{~kg}$ \\
\hline Max. horizontal resistive force & $13 \mathrm{~N} @ \mathrm{dia}=65 \mathrm{~mm}$ \\
\hline Total: finger + support base & $180 \mathrm{~g}$ \\
\hline Diameter smallest graspable obj. & $14 \mathrm{~mm}$ \\
\hline \multicolumn{2}{|l|}{ The DSSF performances } \\
\hline Max. Force at fingertip & $15 \mathrm{~N}$ \\
\hline Max. payload & $4.87 \mathrm{~kg}$ \\
\hline Max. horizontal resistive force & $26 \mathrm{~N} @ \mathrm{dia}=65 \mathrm{~mm}$ \\
\hline Total: finger + support base & $230 \mathrm{~g}$ \\
\hline Diameter smallest graspable obj. & $17 \mathrm{~mm}$ \\
\hline
\end{tabular}
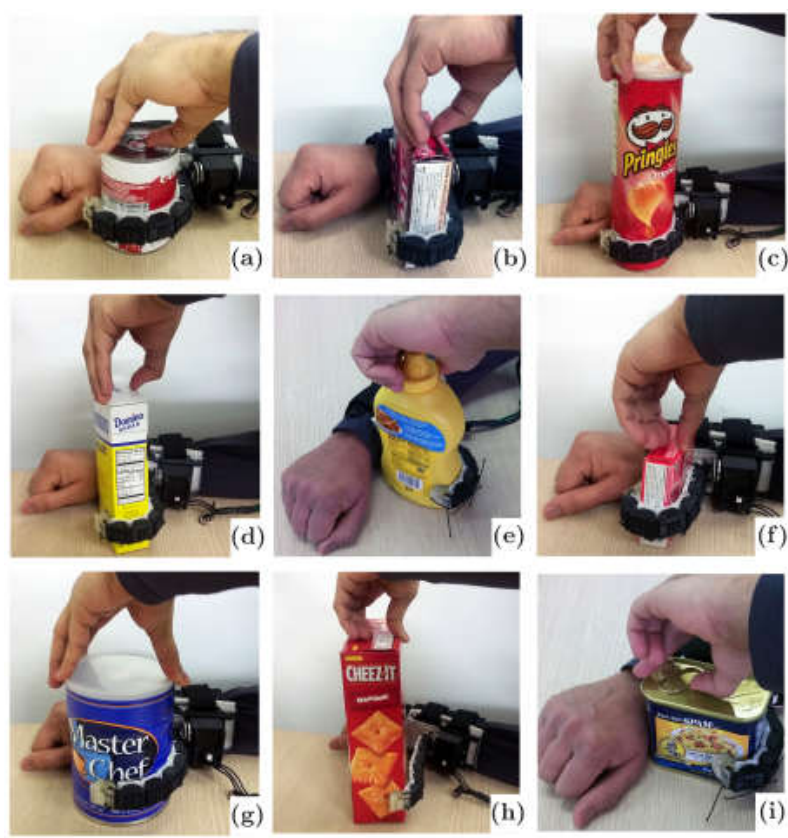

Figure 10. The soft sixth finger grasping various objects with different shapes and sizes: (a) tomato can, (b) chocolate pudding box, (c) chips can, (d) sugar box (e) mustard container, (f) gelatin box (g) coffee can (h) biscuit box (i) meat can. The device is intrinsically-compliant and adopts itself to the shape of the grasped object.

double soft sixth finger shares with the soft sixth finger the same principle design guidelines related to wearability, modularity, symmetrical structure and underactuation. It is composed by two parts: a support base that allows the finger to be worn at the patient forcarm and two fingers.

We fixed the fingers in a " $V$ " configuration. The basic idea bchind setting the two fingers in this configuration was to keep minimum distance at the base of the fingers while maximizing the fingertips' distance at fully extended position with the given length of the finger. The rationale between this choice is the attempt to maximize the distance of the contact points at the fingertips when grasping relatively big size objects. Thanks to the orientation of the finger at the base, when the fingers keep closing so to grasp smaller size objects, the fingertips of both fingers converge one toward the other, hence minimizing the relative distance between them. Thus, this configuration is effective in grasping bigger as well as smaller size objects.

The exploded view of unit module and complete double soft sixth finger is shown in Figure 11. Two tendon wires (one for each flexible finger) and a single actuator control the motion of the device. One end of each tendon wire is fixed to each fingertip, while the other ends of both tendon wires are attached to a single pulley mounted on the shaft of the actuator (MX-28T). 


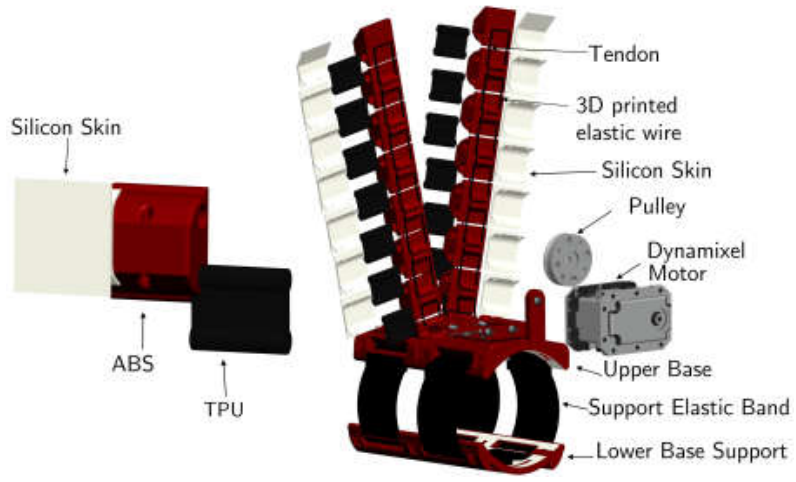

Figure 11. The CAD exploded view of the double soft sixth finger. On the left unit module with single tendon. On the right, the exploded view of complete double soft sixth finger.

When the motor rotates, both tendon wires are wound on the pulley and fingers are flexed to grasp the object. As the motor is rotated in opposite direction, the elastic parts in the joints restore the finger to its extended configuration. The final prototype of the device is shown in Figure 12. Also the double soft sixth finger can be wom on the arm using the base support and the velcro strips. Both fingers can be shaped into bracelet through two separate Dovetail locking mechanism when being not used.

We performed a similar evaluation for the double soft sixth finger to quantify its payload, maximum fingertip force and horizontal grasp resistive force. The results of the experiments are shown in Table 3. The approach of building compensatory robotic device using two robotic fingers improved the payload and horizontal grasp resistive force which in turn can handle relatively heavier objects. The shape adaptation to the grasped object was also confirmed by using the objects in the YCB toolkit.

\subsection{Wearability and device positioning at forearm}

The actuator's controller and battery have been enclosed in a small box to be worn on the patient's belt. Only the device and its actuator are placed at the patient's forearm to keep minimum weight on it. The device can be worn by the user without any assistance, just inserting their hand/arm between the two base parts. Then, using the velcro elastic band, the patient can tight the device at the forearm. The robotic devices can be wrapped up on the arm as bracelets to reduce the encumbrance when being not used as shown in Figures. 8-b and 12-b. The patient can use his or her healthy hand to switch from the rest to the working position and viceversa. The switching between the two positions is achieved through a passive rotatable Dovetail triangular

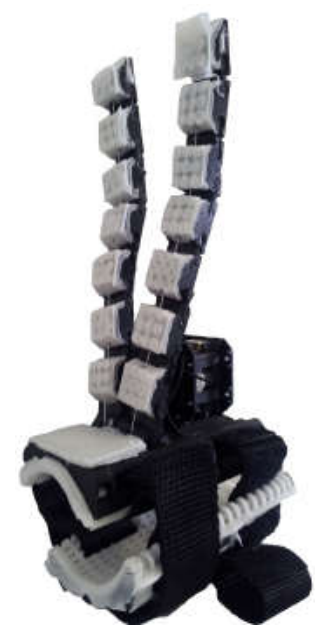

(a) Final prototype of double (b) The double soft sixth soft sixth finger .

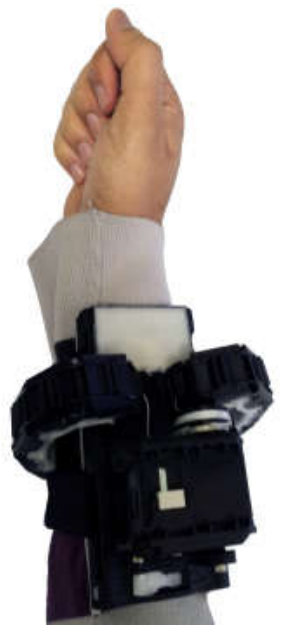

finger in bracelet position.
Figure 12. The final prototype of double soft sixth finger, the device can be worn on the paretic arm through support base and elastic straps. It can be shaped into bracelet when being not used.

locking mechanism. The mechanism consists of two parts, namely A and B. Part A is embedded on the support base while part B is contained in the finger starting module. After coupling both parts together, a pin joint has been added in the center of both parts to allow only rotation while constraining the decoupling of both parts without unscrewing the pin joint. The mechanism has two locking positions. The locking positions are set at the working and rest positions of the extra fingers. Apart from wearability and crgonomics, the device position at the forcarm plays an important role in the task performance.

The location of the device depends on the patient conditions and on the residual mobility of the arm/hand. The compensatory robotic devices can be worn on the distal part of the forcarm (near or on the wrist), so to obtain the grasp by opposing the device to the paretic hand. However, the distal position of the robotic finger may fail when the motor deficit is so advanced that a pathological synergism in flexion has taken place. In this case, the wrist and fingers are too much flexed not allowing successful grasping. When this pathological condition occurs, the extra-finger may be positioned more proximal at the forearm, so to let the grasp be achieved by the extra-fingers opposition to the radial part of the wrist, see Figure 13. This flexibility in the positioning is achieved thanks to the symmetrical structure and the ergonomics of the support base. The support base of the fingers can be translated or rotated along the forearm to place the finger on a suitable orientation. These features enable 

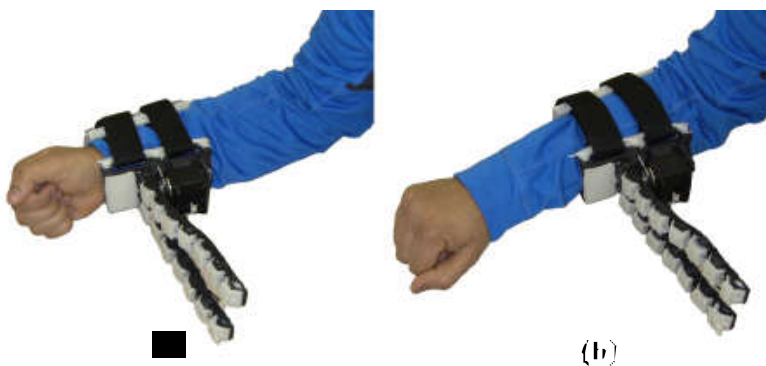

(1)

Figure 13. Two possible positions of grasp compensatory devices at the forearm: (a) the grasp is obtained at hand level by position the device at wrist position, (b) the grasp is obtained at forearm level by position the device near to elbow. The ergonomics design of support base allows the device to adapt patient's condition to obtain grasp at hand or forearm level.

the device to adapt the patient conditions and increases the versatility of the device. Moreover, the elastic straps along with velcro enable the devices to fit to different size of arms and facilitates the patients in wearing robotic device himself without any assistance.

\section{The control interface}

The control interface for patient oriented devices must be intuitive and simple, since chronic patients may also be affected by some cognitive deficits, possibly limiting their compliance during a demanding learning phase. Coordinating the motion of the extra fingers with that of the hand where the devices are worn as presented in $\mathrm{Wu}$ and Asada (2016); Prattichizzo et al. (2014b), is not suitable to patients with hemiparetic upper limb since they are not able to control their hand motion. A possible solution could be the involvement of the controlateral hand in the control process. In Ort et al. (2015) a control strategy that maps the motion of the functional hand onto supernumerary robotic fingers is presented. $\Lambda \mathrm{n}$ instrumented glove is worn on the healthy hand so to track its motion, while a mapping strategy is used to compute the motion of the robotic extra fingers. In Hussain et al. (2015a,b) a ring cmbedding a push-button to control the motion of extra robotic finger is proposed. The ring is worn on the healthy hand so to let the user activate it when necessary. However, preliminary experiments with patients revealed that these solutions limit the mobility and dexterity of the non paretic hand and can also cause possible accidental activation of the device during ADL. Patients also confirmed their preference of having always the healthy hand free during our preliminary tests.

To cope with this issue, we propose the eCap: an Electromyography (EMG) based wireless interface
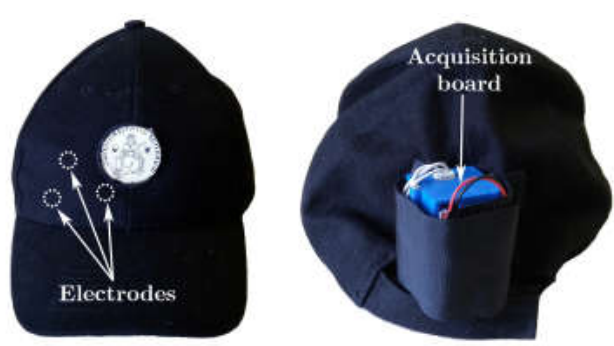

Figure 14. The eCap interface. The EMG electrodes are placed inside the cap at front side to be positioned on the patient's forehead. The acquisition board is placed in a box on the back of the cap.

which maintains the principle of simplicity of a switch without interrupting the patient activities and without the involvement of healthy hand during task execution.

The cCap is a wcarable wircless EMG interface where clectrodes, acquisition and signal conditioning boards are cmbedded in a cap, sec Figure 14. A preliminary version of the control interface has been presented in Hussain ct al. (2016).

This solution allows the patients to autonomously wear the interface using only their healthy hand. Several EMG interfaces have been already successfully adopted for the control of prosthesis Zecca et al. (2002) and exoskeletons Kiguchi et al. (2004). The electrodes are usually placed either in the muscles coupled with the robot (exoskeleton) or in muscles where amputees still have the phantom of functions and hence they are able to generate a repeatable EMG pattern corresponding to each of the functions (prosthesis). For chronic patients it is generally difficult to generate repeatable EMG patterns in their paretic upper limb due to the weakness in muscle contraction control. For this reason, we coupled the flexion/extension motion of the robotic device with the contraction of the frontalis muscle. This muscle is always spared in case of a motor stroke cither of the left or of the right hemisphere due to its bilateral cortical representation. The user can contract this muscle by moving the eycbrows upwards. The electrodes in the cCap capture the arising EMG signal that is acquired through an EMG signal conditioning circuit and processed by a control algorithm as explained in the following.

EMG measures the electrical potential between a ground electrode and a sensor electrode. It is possible to measure signals either within the muscle (invasive EMG) or on the skin above a muscle (surface EMG) Saponas et al. (2008).

We used surface EMG electrodes to measure electrical signals associated with the patient's frontalis muscle. In particular, on the inner side of the eCap, we installed non-gelled reusable silver/silver-chloride electrodes, as they 
present the lowest noise interface and are recommended for biopotentials recording Merletti ct al. (2009). We designed an EMG signal acquisition board taking into consideration the requirements associated with bandwidth, dynamic range and physiological principles. The typical EMG waveform is characterized with a spectral content between 10 to $250 \mathrm{~Hz}$ with amplitude up to $5 \mathrm{mV}$, depending on the particular muscle Merlo and Campanini (2010).

Figure 15 shows the block diagram of the implemented EMG circuit board. Three electrodes are interfaced to the board: two of them $\left(V_{I N}\right.$, and $\left.V_{I N}\right)$ are connected to the inputs of an instrumentation amplificr (In-Amp), while the third one called "ground electrode" is connected to a midsupply reference voltage $\left(V_{s s}=V_{c c} / 2\right)$. This configuration improves the quality of EMG signal acquisition as it increases the common mode rejection ratio (CMRR).

The first stage of the EMG board is an In- $\Lambda \mathrm{mp}$ with an additional stage of $\Lambda \mathrm{C}$-coupling. This configuration allows a precise control of DC levels rejecting undesired DC offset voltage introduced by electrode-skin interface. The DC component is subtracted by feeding the output signal back to the reference input of the In-Amp, by an integrator fecdback network, which results in a first-order highpass response. The second stage of the EMG board is a $4^{\text {th }}$ order lowpass Butterworth filter. An active topology (a Sallen-Key circuit implementation $-4^{\text {th }}$ order low-pass filter cascading two stages of $2^{\text {nd }}$ order) was chosen to get a better performance and less complexity than a passive one. The specifications of the EMG acquisition board are summarized in Table 4.

The acquired EMG signal is sampled at $1 \mathrm{kHz}$ (double EMG band) to avoid aliasing and a wircless communication is realized by a pair of Xbec modules (Scrics 1). The transmitter is embedded in the eCap while the receiver is placed on the actuator controller unit. The reference value of

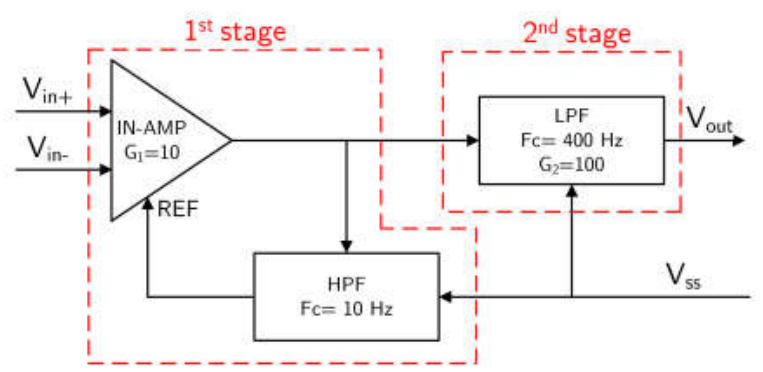

Figure 15. Block diagram of the EMG circuit board. $V_{I N-}$ and $V_{I N}$ are the "detecting electrodes" while $V_{s s}=V_{c c} / 2$ is the "ground electrode". $R E F$ is the reference voltage terminal of the In-Amp.

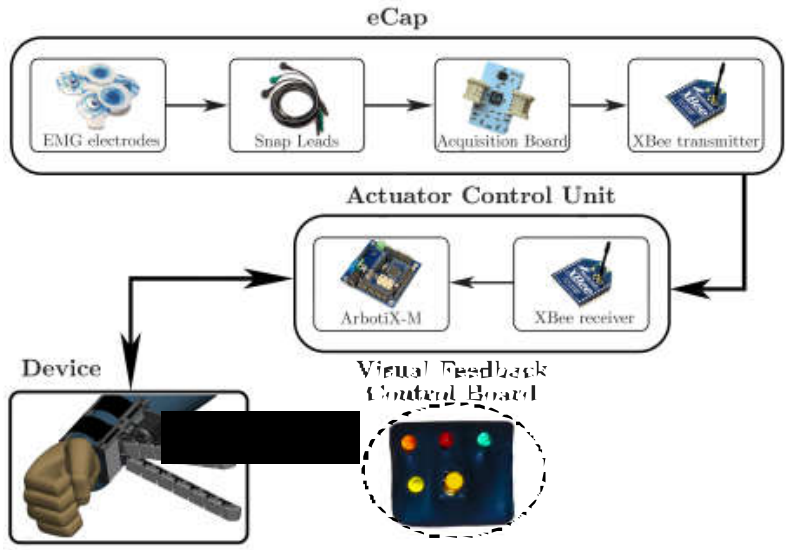

Figure 16. The block diagram of EMG wireless and push-button interface with actuator control unit. LEDs associated to the motion of device are mounted on visual feedback control board.

Table 4. Specifications of EMG Acquisition Board

\begin{tabular}{|l|c|}
\hline EMG acquisition box dimensions & $35 \times 31 \times 15 \mathrm{~mm}^{3}$ \\
\hline EMG acquisition box weight & $16 \mathrm{~g}$ \\
\hline Principle & Differential voltage \\
\hline Number of electrodes & 3 \\
\hline Bandwidth & $10-400 \mathrm{~Hz}$ \\
\hline Gain & 1000 \\
\hline Input Impedance & $100 \mathrm{G} \Omega$ \\
\hline CMRR & $110 \mathrm{~dB}$ \\
\hline Operating voltage & $V c c=3.3 \mathrm{~V}$ \\
\hline
\end{tabular}

the received EMG signals were normalized using maximum voluntary contraction (MVC) technique Farina and Merletti (2000). This solution avoids the problems related to the high influence of detection condition on EMG signal amplitude. In fact, amplitude can greatly vary between electrode sites, subjects and even day to day measures of the same muscle site. We implemented an auto-tuning procedure based on the MVC in order to better match the user-dependent nature of the EMG signal. This is the major improvement with respect to the eCap version proposed in Hussain et al. (2016) where the sensitivity of the system was manually set through a potentiometer. The implemented MVC routine consists of a 3 s time window in which the users slowly start increasing the contraction of the forchead muscle to reach their maximum effort. The MVC value itself is not calculated as a single peak data point to avoid high variability. In order to obtain a more stable reference value, we have implemented an algorithm using a sliding window technique of $500 \mathrm{~ms}$ duration to compute the mean amplitude of the highest signal portion acquired during the $3 \mathrm{~s}$ time window.

The motion of compensatory robotic device is then controlled by using a trigger signal based finite state machine 

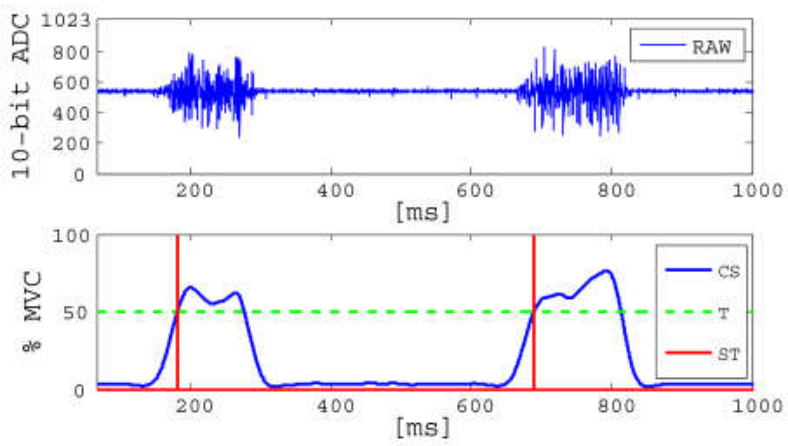

Figure 17. Top: raw EMG signal. Bottom: example of two activations in a time window of $1 \mathrm{~s}$. CS (blue) is the processed EMG signal after the operations of rectification, normalization and filtering; $T$ (green) is the threshold and $S T$ (red) is the resulting trigger.

(FSM) Oskoei and $\mathrm{Hu}$ (2007). The trigger signal is obtained by using a single-threshold value defined as the $50 \%$ of the $\mathrm{MVC}$, a level that was repeatable and sustainable for the subject without producing undue fatigue during the use of the device. We set a minimum time $(20 \mathrm{~ms})$ in which the EMG signal has to constantly stay over the threshold to generate the trigger signal to prevent false activation due to glitches or to spontaneous spikes. Figure 17 shows the raw EMG signal and the signal after the conditioning operations, i.e., rectification, normalization and filtering. The red signal shown in bottom graph of Figure 17 is the resultant trigger signal which is generated if the EMG signal exceeds the threshold. The outputs of the FSM are predefined commands based on sequences of input signals. We consider a finite number of states, transition between those states, and commands. States represent predefined motion commands for the robotic device and transition actions are associated with contractions of the frontalis muscle. The patients control the motion/stop of the finger with a single muscle contraction (cvent $e_{1}$ ). Once the finger is stopped, two contractions (event $e_{2}$ ) in a time window of $1 \mathrm{~s} \mathrm{switch}$ the direction of motion from flexion to extension and viceversa. The time window length was experimentally selected after the repeated trials with patients and resulted to be in-line with the one proposed in Felzer and Freisleben (2002). A software defined trigger (event $e_{4}$ ) stops the actuator's motion once the object is considered as grasped, to avoid a torque overloading situation. The grasp confirmation is detected by continuous monitoring the actuator's shaft position and the exerted torque. During the grasping procedure, if the position does not change in a time window of $2 \mathrm{~s}$ and a predefined torque threshold is reached, the object is considered as grasped. The proposed FSM is reporled in Figure 18.

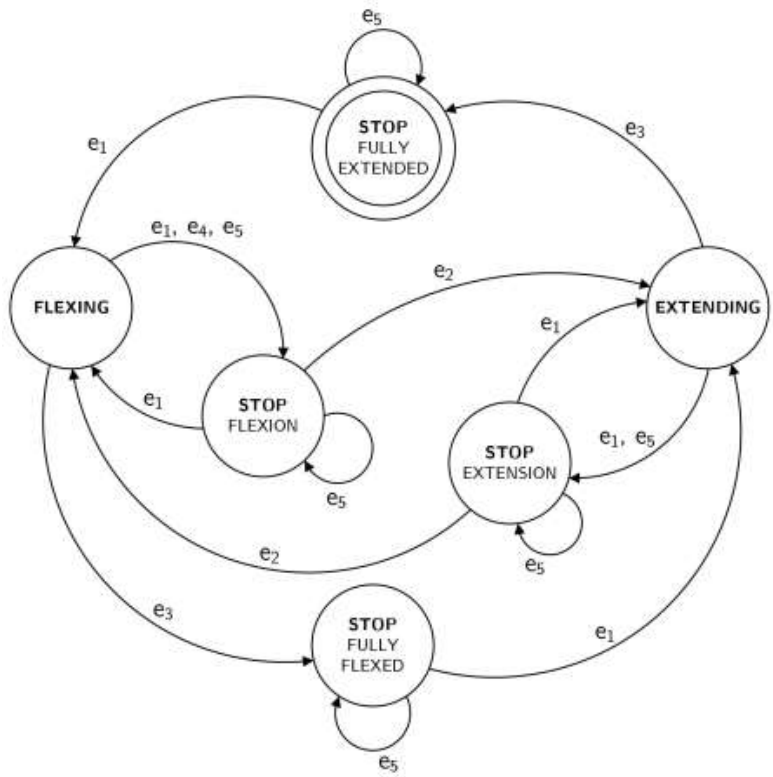

Figure 18. The proposed finite state machine (fsm) for the motion control of the robotic devices. Events $e_{1}$ and $e_{2}$ are generated by the user, while $e_{3}$ is a software defined event. Event $e_{1}$ occurs once the object is grasped. Event $e_{5}$ activates on switching between two proposed control interfaces (eCap or push-button).

A LED board (see Figure 16) is used to provide a visual feedback of the selected commands. In particular, a yellow LED blinks on each trigger signal. When flexion is selected an orange LED is turn on, while a green LED shows the extension. Finally a red LED is turn on when the device is stopped. At this stage, the LED associated to the previous selected state is also turned on to remind the user about the last stage of the device. To provide an additional interface for the user, as well as a recovery mode for possible problem in the cCap communication, we added a push-button on the LED board as further possible control.

Both interfaces use the same trigger-based logical scheme to control the motion of robotic devices. The switching between two control interfaces can be achieved at any moment by a toggle switch installed on the controller board. If the eCap is selected, the procedure of maximum voluntary contraction (MVC) is first executed. Once it is completed, the program passes from calibration to test mode. If the pushbutton is selected, the control algorithm directly jump to the test mode. In test mode, the user tests the selected interface by displaying the output on the LEDs mounted on the LED board to get familiar with the interface without using the robotic device. When the eCap control interface is selected, the user can do the MVC calibration again (if needed) by simply pressing once the push-button. If the test mode output confirms to the expected program behaviour and user is ready 


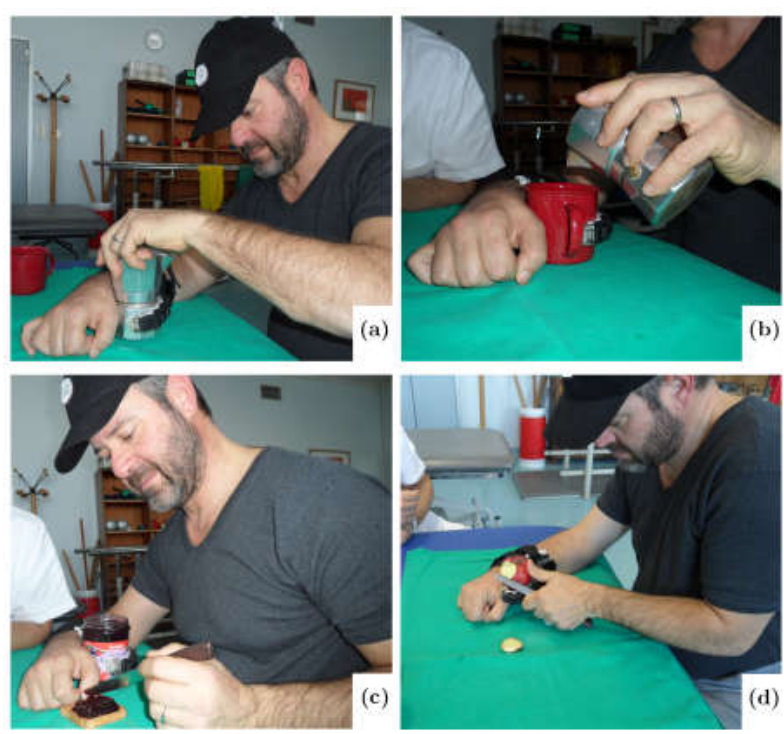

Figure 19. Preparing breakfast with the help of grasping compensatory tool.(a) opening the Moka pot, (b) pouring coffee into cup, (c) spreading jam on bread, (d) peeling apple

to use the device, he or she can switch to the device mode, where, motion of robotic device is controlled according to FSM in Figure 18. The switching from test mode to device mode is achicved by pressing the push-button for more than one second and is represented in the FSM in Figure 18 by the event $c_{5}$. The process repeats every time the user switch to the other control interface.

\section{Tests with chronic stroke patients}

We performed a series of experiments with five chronic stroke patients (four malc, onc female, age $10-62$ ) to prove the effectiveness of the devices in grasp compensation. Written informed consent was obtained from all participants. The procedures were in accordance with the Declaration of Helsinki. We targeted ADL bi-manual tasks to cvaluate if the compensatory robotic devices can assist the patients. In order to use the proposed devices, the subjects should have residual mobility of the arm. For being included in the experimental phase, patients had to score $<2$ when their motor function was tested with the National Institute of Health Stroke Scale (NIHSS) Brott et al. (1989), item 5 "paretic arm". Moreover, the patients had to show the following characteristics: normal consciousness (NIHSS, item 1a, 1b, 1c =0), absence of conjugate eyes deviation (NIHSS, item $2=0$ ), absence of complete hemianopia (NIHSS, item $3 \leq 1$ ), absence of ataxia (NIHSS, item $7=0$ ), absence of completely sensory loss (NIHSS, item $8 \leq 1$ ), absence of aphasia (NIHSS, item $9=0$ ), absence of profound extinction and inattention (NIIISS, item $11 \leq 1$ ).

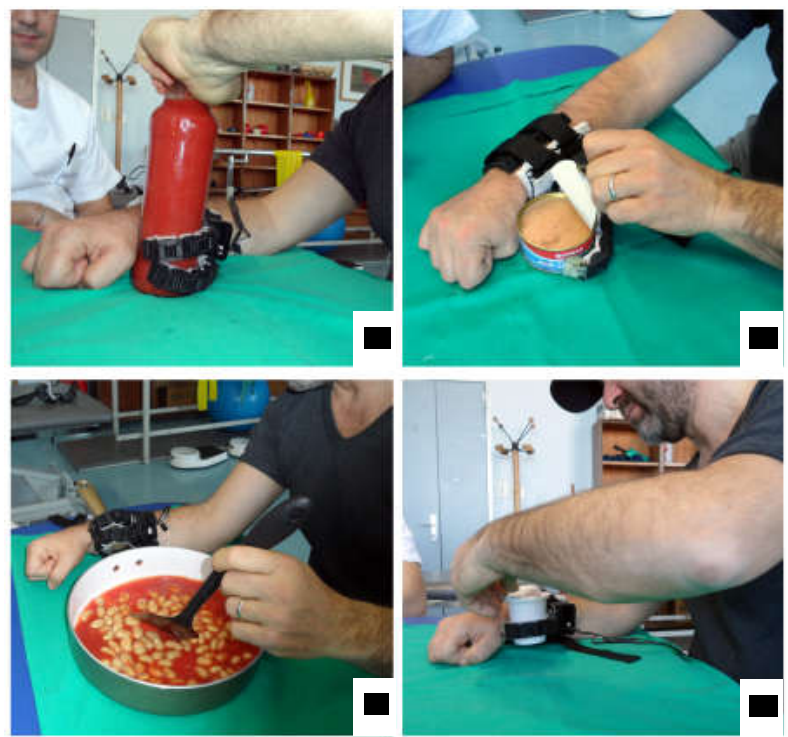

Figure 20. Preparing lunch with the help of grasping compensatory robotic device. (a) opening tomato sauce jar, (b) opening tuna can, (c) stirring food, (d) opening yogurt cup

The goal of the tests was to evaluate how quickly the patients could learn to use the devices and which device they preferred to fulfill a certain task. The patients were asked to select between the soft sixth finger and the double soft sixth finger to perform the proposed tasks. We recorded the time to complete the task (T) in seconds (s) for each task. Patients wore the robotic device on their paretic limb, on the left for two subjects and on the right one for the other three. The rehabilitation team assisted the subjects during a training phase that lasted about one hour. During this phase, the optimal position of the device on the arm was evaluated according to the patient motor deficit. After the training phase, the subjects were asked to perform a list of bimanual tasks with the aid of proposed devices. We proposed to the patients three possible scenarios. The first included two different kitchen activities involving multiple bi-manual tasks. In the second, we tested the device with bi-manual tasks using tools. Finally, we tested the use of the robotic extra fingers to carry a shopping bag while walking.

\subsection{Kitchen Scenario}

Cooking in the kitchen involves a variety of bi-manual tasks and many of them are based on hold and manipulate techniques. The compensatory robotic devices can support the patients in performing such tasks even if one hand is paretic. Figure 19 and Figure 20 show the snapshots of the tasks performed by the subjects to prepare the breakfast and lunch. 

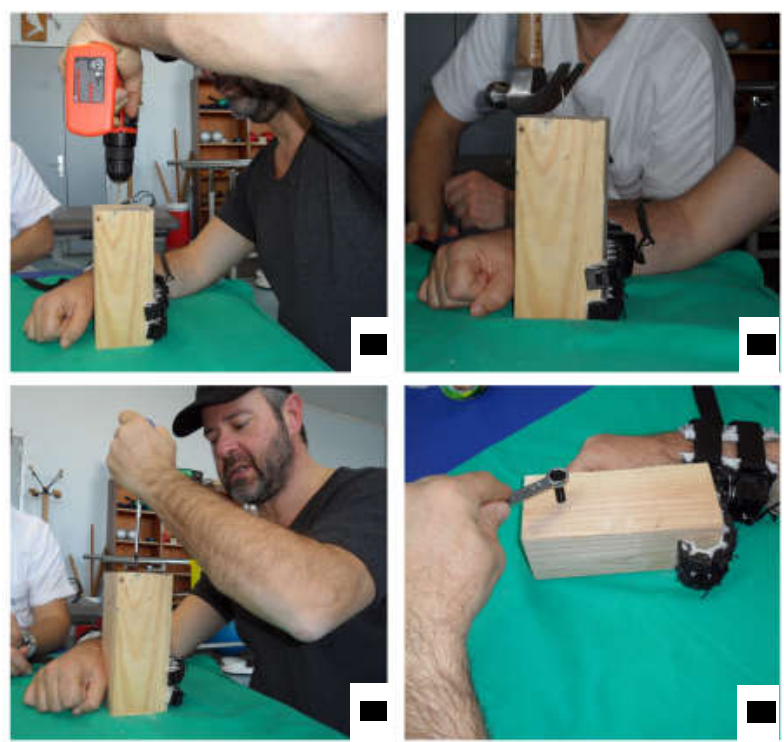

Figure 21. Using different tools in bi-manual tasks with the aid of robotic device. (a) drilling in wood block, (b) removing nail using claw hammer (c) inserting tapcon screw (d) tightening or loosening bolt

\section{Preparing breakfast}

We asked the patients to simulate the activities of preparing coffee, putting the jam on a bread slice and peeling an apple for breakfast.

Task 1 "Opening the coffee pot": hold firmly the base of coffee moka pot with the help of robotic device while using the healthy hand to unscrew the upper part (sec, Figure 19-a). Task 2 "Closing the coffee pot": fill the filter with a mixture of coffec grounds. Grasp the base part with the device and close the pot again.

Task 3 "Pouring colfee into cup": pour coffcc using the healthy hand while holding cup with the compensatory device, see Figure 19-b.

Task 4 "Opening jam jar": grasp the jar with the device and non-functional arm while opening the cap with the functional one.

Task 5 "Spreading jam on bread": grasp the jam jar to take jam from it by holding the knife in healthy hand. Spread jam on a bread slice using the functional hand, see Figure 19-c. Task 6 "Peeling apple": grasp the apple with device and peel it using a knife in the healthy hand, see Figure 19-d.

All the patients choose the soft sixth finger to perform the tasks, since the single finger results are more suitable while manipulating relatively light weight and smaller objects. Results on time to complete and success rate are reported in Table 5.

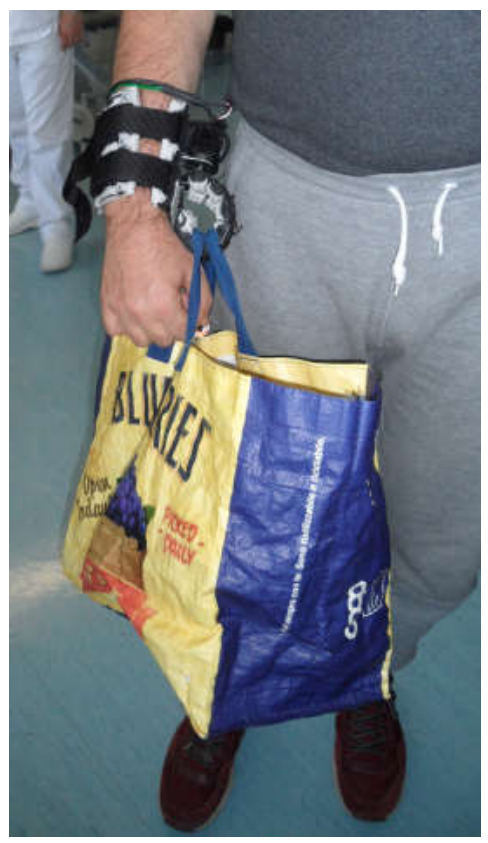

Figure 22. Carrying shopping bag with the help of grasping compensatory robotic device.

\section{Preparing lunch}

Task 1 "Opening tomato sauce jar": constrain the motion of the tomato jar with the device and the paretic arm while the healthy hand unscrews its cap, see Figure 20-a.

Task 2 "Pouring": pouring the tomato sauce from its jar into a cooking pot.

Task 3 “Opening tuna can': hold the tuna can with the device while the functional hand opens its cap, sec Figure 20-b.

Task 4 "Opening beans can": constrain the motion of a beans can with the compensatory robotic device and the paretic arm while the healthy hand opens its cap.

Task 5 "Pouring": pouring the beans from the can into a cooking pot.

Task 6 "Stirring": hold firmly the cooking pot with the compensatory robotic device while the functional hand stirs the food in it, see Figure 20-c.

Task 7 "Opening yogurt cup": constrain the yogurt cup and remove its cover, see Figure 20-d.

Nll patients decided to use the double soft sixth finger to perform tasks (a) and (c), while the soft sixth finger was sclected to complete tasks (b) and (d). Snapshots of the execution of the tasks are reported in Figure 20. Results are reported in Table 5.

\subsection{Tools Activities}

Tools activities are another example of ADL where many tasks are based on a hold and manipulate principle. The presence of the compensatory robotic device can help the 
patient to complete such bi-manual tasks even if one hand is non-functional. We asked the patients to use the tools to perform the following tasks.

Task 1: "Drilling in wood block": grasp the wood block with the device and impaired arm. Drill a hole in the wood block while using the drill in the healthy hand, see, Figure 21-a.

Task 2: "Removing nail using claw hammer": hold firmly the wood block with the compensatory robotic device and the paretic arm. Use a claw-hammer in the healthy hand to pull the nail from the wood block, sce, Figure 21-b.

Task 3: "Inserting tapcon screw in the drilled hole": use the device and the paretic arm to hold the object with a drilled hole. Place the tapping screw at the hole position, use the screw driver with the functional hand to screw it until it is completely inserted in the hole, see Figure 21-c.

Task 4: "Tightening or loosening bolt using wrench key": constrain the object with the device and the paretic arm and use the wrench key in healthy hand to tight or loose the bolt, see Figure 21-d.

All patients selected the double soft sixth finger to perform the tasks. Results are reported in Table 5.

\subsection{Active Hook}

The last application proposed was to carry a shopping bag with the compensatory robotic device while walking as shown in Figure 22. The patients were successfully able to carry the bag using the robotic devices on paretic arm. As expected, the double soft sixth finger was able to carry heavier bag as compared to the soft sixth finger due to its higher payload. In particular, all the patients were able to carry a bag of $1.5 \mathrm{~kg}$ using the soft sixth finger and a bag of $3 \mathrm{~kg}$ using the double finger version.

Table 5. Mean times to complete ( $T$ ) and success rates (SR) for the proposed tasks.

\begin{tabular}{|c|c|c|c|c|c|c|}
\hline & \multicolumn{2}{|c|}{ Breakfast } & \multicolumn{2}{c|}{ Lunch } & \multicolumn{2}{c|}{ Tools } \\
\hline & $\mathrm{T}$ & $\mathrm{SR}$ & $\mathrm{T}$ & $\mathrm{SR}$ & $\mathrm{T}$ & $\mathrm{SR}$ \\
\hline Task 1 & $13 \mathrm{~s}$ & $100 \%$ & $\mathbf{9}$ & $\mathbf{1 0 0 \%}$ & $15 \mathrm{~s}$ & $100 \%$ \\
\hline Task 2 & $\mathbf{7 s}$ & $100 \%$ & $11 \mathrm{~s}$ & $\mathbf{8 0} \%$ & $16 \mathrm{~s}$ & $100 \%$ \\
\hline Task 3 & $4 \mathrm{~s}$ & $80 \%$ & $12 \mathrm{~s}$ & $\mathbf{1 0 0} \%$ & $20 \mathrm{~s}$ & $100 \%$ \\
\hline Task 4 & $8 \mathrm{~s}$ & $100 \%$ & $10 \mathrm{~s}$ & $\mathbf{1 0 0 \%}$ & $\mathbf{2 1} \mathrm{s}$ & $\mathbf{8 0} \%$ \\
\hline Task 5 & $13 \mathrm{~s}$ & $80 \%$ & $12 \mathrm{~s}$ & $\mathbf{6 0} \%$ & & \\
\hline Task 6 & $18 \mathrm{~s}$ & $100 \%$ & $\mathbf{1 4} \mathrm{s}$ & $\mathbf{1 0 0 \%}$ & & \\
\hline Task 7 & & & $15 \mathrm{~s}$ & $\mathbf{1 0 0 \%}$ & & \\
\hline
\end{tabular}

\subsection{Questionnaires}

After the experiments, we asked the patients about the usefulness and possible concerns related to the compensatory robotic devices for performing $\Lambda \mathrm{DL}$ tasks. The patients were asked to fill the Uscfulness-Satisfaction-and-Ease-ofuse questionnaire (USE, Lund (2001)) that focuses on the experience of the system usage. This questionnaire uses a seven-point Likert rating scale. Mean and standard deviation (SD) of the questionnairc factors are presented in Table 6.

Table 6. Questionnaire factors and relative marks. The mark ranges from " 1 - strongly disagree" to "7 - strongly agree". Mean and standard deviation (Mean (SD)) are reported.

\begin{tabular}{|l|c|}
\hline Questionnaire factors & Mean (SD) \\
\hline \hline Usefulness & $5.6(0.8)$ \\
\hline Ease of use & $6.0(0.6)$ \\
\hline Ease of learning & $6.5(0.8)$ \\
\hline Satisfaction & $5.8(0.7)$ \\
\hline
\end{tabular}

Moreover, to evaluate the patient's satisfaction to the proposed compensatory devices and their leatures, we asked the patients to fill the first part of the QLEST 2.0 questionnaire Demers et al. (2002). The purpose of the QUEST questionnaire is to evaluate how satisfied patients are with the proposed assistive device. The mark ranges from " $1=$ not satisfied at all" to " $5=$ very satisfied". Mean and standard deviation (Mean (SD)) are reported in Table 7.

Table 7. Quebec User Evaluation of Satisfaction with assistive Technology. The mark ranges from " 1 - not satisfied at all" to " $\mathrm{\nu}$ = very satisfied". Mean and standard deviation (Mean (SD)) are reported.

\begin{tabular}{|l|c|}
\hline How satisfied are you with & Mean (SD) \\
\hline $\begin{array}{l}\text { the dimensions (size, height, length, } \\
\text { width) of your assistive device? }\end{array}$ & $1.6(0.8)$ \\
\hline the weight of your assistive device? & $4.0(0.6)$ \\
\hline $\begin{array}{l}\text { how safe and sccure your assistive device } \\
\text { is? }\end{array}$ & $4.5(0.8)$ \\
\hline $\begin{array}{l}\text { the durability (endurance, resistance to } \\
\text { wear) of your assistive device? }\end{array}$ & $4.6(0.7)$ \\
\hline how easy it is to use your assistive device? & $1.6(0.7)$ \\
\hline \begin{tabular}{l} 
how comfortable your assistive device is? \\
\hline $\begin{array}{l}\text { how effective your assistive device is (the } \\
\text { degree to which your device meets your } \\
\text { needs)? }\end{array}$
\end{tabular} & $1.2(0.7)$ \\
\hline
\end{tabular}

\section{Results and discussion}

The ergonomics and functional requirements listed in Table 1 have been considered in the design and development of the 
robotic devices. Table 8 summarizes the devices achieved requirements.

The robotic extra fingers have been tested with different targets in order to demonstrate how the soft fingers can adapt to the shape of the object, producing a stable enveloping grasp. We tested the device with five chronic stroke patients in ADL. The proposed robotic devices successfully enabled the patients to complete the presented bi-manual tasks. Failure in tasks' fulfillment reported in Table 5 are mainly due to the weakness of the arm motion of one patient. The experiments authenticated that the presented robotic devices can be an effective aid for chronic stroke patients to perform simple ADI tasks. The patients' questionnaire feedback showed the effectiveness of the proposed compensatory robotic devices in assisting $\Lambda D L$ tasks.

The proposed robotic devices were an effective aid in completing the ADL bi-manual tasks. If compared to the old versions of the device, the soft sixth finger showed better performance due to the new actuation, the more stable support base and the increased friction at contact points. The realization of a new device, the double soft sixth finger, increased the potential use of compensatory devices in the ADL tasks, since it is able to realize a more stable grasp in relatively more payload demanding and pouring tasks. At this carly stage of rescarch, we cannot determine which device is better to fulfill a certain task. The soft sixth finger was more casy to usc when it is necessary to grasp small objects. It is also more wearable and portable with respect to the version with two fingers. On the other hand, the double soft sixth finger has a higher payload and can be used in more demanding manipulation tasks, such as pouring water from a bottle. This improvement in grasp stability comes at the cost of a higher weight and a reduced wearability. We believe it is worth exploring both the solutions in different applications and different tasks, and to keep developing the two platforms in collaboration with the clinicians and the patients.

Although the experiments with patients showed the effectiveness of the devices in the completion of some ADL, at the moment this approach has some limitations. Patients suffering from hemiplegia or hemiparesis can vary over a wide range, from mild weakness and loss of dexterity in the fingers to complete paralysis in the left or right side of the body. Even if the proposed compensatory devices are able to compensate in terms of grasping, the use of the device requires some mobility in the impaired arm with even non-functional hand. Complex manipulation bi-manual tasks like tying shoelaces or buttoning, are too demanding and out of scope of both the selected group of patients and current devices. However, many ADL tasks including those presented in the paper, can successfully be completed with the aid of proposed robotic devices .

\section{Conclusion and future perspective}

This paper presents design, analysis, manufacturing, experimental characterization and evaluation of two prototypes of robotic extra fingers that can be used as grasp compensatory robotic devices for hemiparetic upper limb. We tested the devices with chronic stroke patients through qualitative experiments based on ADL.

Currently, we are investigating whether it is possible to introduce the robotic extra fingers in an carly rehabilitation phase. Many of the rchabilitation strategies involve ADL tasks where patients attempt to make use of weak hand even though it is not functionally capable. This can result in feclings of frustration. Presenting an active compensatory tool may help in the initial phase to promote the use of the arm even if the hand grasp function is not recovered. In a recent work Hussain et al. (2017b), we presented the combination of supernumerary robotic finger with a robotic arm support that can be used as an assistive device to support the patient arm weight. The overall proposed system can provide the needed assistance during paretic upper limb rehabilitation involving both grasping and arm mobility to solve task-oriented activities.

Finally, we are investigating the possibility of using our devices in patients affected by other neurological discases possibly affecting hand grasping, such as Multiple Sclerosis, Amyotrophic Lateral Sclerosis and paresis duc to cervical spinal cord lesions.

\section{Appendix A: Kinematics Modeling}

In order to find the global coordinates of each module, we need to add the contributions from previous relative deflections and account for rotation due to the angle of the previous module. Homogeneous transformation matrices can be used to perform this operation. Let us consider a point described by $\vec{p}_{i}$ that we want to rotate about another point $\vec{p}_{r}$ by an angle $\alpha$ to obtain the point's coordinates in a global frame. Using the standard procedure, we first translate the point $\vec{p}_{r}$ back to the origin, perform the rotation and translate back to $p_{r}^{\prime}$. The sequence of transformation is as follows

$$
\vec{p}_{f}-T_{1}\left(\vec{p}_{r}\right) C(\alpha) T_{2}\left(\vec{p}_{r}\right) \cdot \vec{p}_{i}
$$

Where, $\vec{p}_{f}$ is the point's coordinates in the global frame. $T_{1}\left(\overrightarrow{p_{r}}\right)$ and $T_{2}\left(\overrightarrow{p_{r}}\right)$ are the transformation matrices dependent 
Table 8. How the proposed robotic devices meet the ergonomics and functional requirements listed in Table 1

\begin{tabular}{|c|c|c|}
\hline Category & Requirement & Actual realization \\
\hline \multicolumn{3}{|c|}{ Ergonomics } \\
\hline & Extreme Wearability & $\begin{array}{l}\text { The devices can be worn through support base and can be } \\
\text { shaped into bracelet when being not used. }\end{array}$ \\
\hline & Portability & $\begin{array}{l}\text { Portable complete system including power supply and } \\
\text { actuator control unit. The wircless communication } \\
\text { between eCap and robotic device. }\end{array}$ \\
\hline & Weight & Maximum $230 \mathrm{~g}$ at arm (see, table 7 ). \\
\hline & Ease of use & $\begin{array}{l}\text { Ease in wearing is realized by eCap, support base and } \\
\text { elastic straps. Easy working principle and few control } \\
\text { inputs from user (see, table } 6 \text { ). }\end{array}$ \\
\hline \multicolumn{3}{|l|}{ Functional } \\
\hline & Robustness & $\begin{array}{l}\text { The intrinsic compliance and flexible structure (see, } \\
\text { table 7). }\end{array}$ \\
\hline & $\begin{array}{l}\text { Fatigue avoidance and } \\
\text { salety }\end{array}$ & $\begin{array}{l}\text { No mechanical forces by robotic device on impaired } \\
\text { hand. Light in weight. }\end{array}$ \\
\hline & $\begin{array}{l}\text { Device adaptability to } \\
\text { paticnt }\end{array}$ & $\begin{array}{l}\text { Adaptable in terms of positioning and left/right } \\
\text { hemiparetic upper limb with different sizes. }\end{array}$ \\
\hline & $\begin{array}{l}\text { Control interface adaptabil- } \\
\text { ity to patient }\end{array}$ & $\begin{array}{l}\text { Auto-tuning calibration to better match the user- } \\
\text { dependent nature of the EMG signal. }\end{array}$ \\
\hline & $\begin{array}{l}\text { Device coupling with human } \\
\text { arm }\end{array}$ & $\begin{array}{l}\text { Devices firm grip at forearm by coupling of two rigid } \\
\text { parts of support base having silicon skin to increase } \\
\text { friction and two parallel velcro strap to tight both parts } \\
\text { at the arm. }\end{array}$ \\
\hline & Object shape adaptability & Underactuation and passive compliance. \\
\hline & Mechanical power & Actuator with Controllable mechanical power (torque). \\
\hline & Configurability & $\begin{array}{l}\text { Modular design, easy modules assembly (flexible part } \\
\text { slides in stiff part). Selectable desired control interface, } \\
\text { i.e., EMG or push button. }\end{array}$ \\
\hline & Functional versatility & $\begin{array}{l}\text { Devices functionality ranging from clinical needs to } \\
\text { various indoor and outdoor ADL tasks. }\end{array}$ \\
\hline & Energy efficiency & $\begin{array}{l}\text { Rechargeable light weight portable batteries. Actuator } \\
\text { capability to maintain the stall torque at minimum current } \\
\text { consumption. }\end{array}$ \\
\hline & Error tolerance & $\begin{array}{l}\text { Underactuation, passive compliance and high friction } \\
\text { through silicon skin at possible contact points. }\end{array}$ \\
\hline & $\begin{array}{l}\text { Simple and intuitive inter- } \\
\text { faces }\end{array}$ & $\begin{array}{l}\text { Trigger signal based simple control, LEDs mounted } \\
\text { visual fecdback control board for further intuitive } \\
\text { interface (see, table 6). }\end{array}$ \\
\hline
\end{tabular}

on the intermediate point $p_{r}^{\prime}$ and $C(\alpha)$ is a rotation matrix dependent on the angle $\alpha$. Using equations 1,2 and 13 .

$$
\left[\begin{array}{c}
L_{i} \\
\Delta_{i} \\
1
\end{array}\right]=\left[\begin{array}{ccc}
1 & 0 & L_{i-1} \\
0 & 1 & \Delta_{i-1} \\
0 & 0 & 1
\end{array}\right]\left[\begin{array}{ccc}
\cos \left(\theta_{i-1}\right) & -\sin \left(\theta_{i-1}\right) & 0 \\
\sin \left(\theta_{i-1}\right) & \cos \left(\theta_{i-1}\right) & 0 \\
0 & 0 & 1
\end{array}\right]
$$

$$
\left[\begin{array}{ccc}
1 & 0 & -L_{i-1} \\
0 & 1 & -\Delta_{i-1} \\
0 & 0 & 1
\end{array}\right]\left[\begin{array}{c}
L_{i-1}+l_{i} \\
\Delta_{i-1}+\delta_{i} \\
1
\end{array}\right]
$$

\section{Acknowledgements}

The authors are grateful to Dr. David Cioncoloni, Sabrina Taddei and Prof. Simone Rossi from the Dipartimento di Scienze Mediche, 
Chirurgiche e Neuroscienze, UOC Neurologia e Neurofisiologia Clinica, Brain Investigation and Neuromodulation Lab. (Si-BIN), Siena, Italy, for their suggestions and the help in recruiting and assisting the patient during the experiments.

The research leading to these results has received funding from the European Union's Ilorizon 2020 Research and Innovation Programme under Grant Agreement No 688857 of the project SoftPro: Syncrgy-based Open-source Foundations and Technologies for Prosthetics and Rchabilitation.

\section{References}

Aszmann, O. C., Roche, A. D., Salminger, S., Paternostro-Sluga, T., Herceg, M., Sturma, A., Hofer, C., and Farina, D. (2015). Bionic reconstruction to restore hand function after brachial plexus injury: a case series of three patients. The Lancel, 385(9983):2183-2189.

Balasubramanian, S., Klein, J., and Burdet, E. (2010). Robotassisted rchabilitation of hand function. Current opinion in neurology, 23(6):661-670.

Belter, J. T. and Segil, J. L. (2013). Mechanical design and performance specifications of anthropomorphic prosthetic hands: a revicw. Journal of rehabilitation research and development, 50(5):599.

Bicchi, A., Bavaro, M., Boccadamo, G., De Carli, D., Filippini, R., Grioli, G., Piccigallo, M., Rosi, A., Schiavi, R., Sen, S., ct al. (2008). Physical human-robot interaction: Dependability, safety, and performance. In Advanced Molion Control, 2008. AMC'08. 10th IEEE International Workshop on, pages 9-14. IEEE.

Birglen, L., Lalibertè, T., and Gosselin, C. (2008). Underactuated Robotic Hands, volume 40 of Springer Tracts in Advanced Robotics. Springer.

Brott, T., Adams, H., Olinger, C. P., Marler, J. R., Barsan, W. G., Biller, J., Spilker, J., Holleran, R., Ebcrle, R., and Hertzberg, V. (1989). Measurements of acute cerebral infarction: a clinical cxamination scalc. Stroke, 20(7):864-870.

Çalli, B., Walsman, A., Singh, A., Srinivasa, S., Abbecl, P., and Dollar. $\Lambda$. M. (2015). Benchmarking in manipulation rescarch: The YCB object and model set and benchmarking protocols. CoRR, abs/1502.03143.

Casson, A. J., Logesparan, L., and Rodrigucz-Villegas, E. (2010). An introduction to future truly wearable medical devicesfrom application 10 asic. In Engineering in Medicine and Biology Society (EMBC), 2010 Annual International Conference of the IEEE, pages 3430-3431. IEEE.

Catalano, M. G., Grioli, G., Farnioli, E., Serio, A., Piazza, C., and Bicchi, A. (2014). Adaptive synergies for the design and control of the pisa/iit softhand. The International Journal of Robotics Research, 33(5):768-782.

Davis, W. E. and Burton, A. W. (1991). Ecological task analysis: Translating movement behavior theory into practice. Adapted Physical Activity Quarterly, 8(2):154-177.

Demers, L., Weiss-Lambrou, R., and Ska, B. (2002). The quebec user evaluation of satisfaction with assistive technology (quest 2.0): an overview and recent progress. Technology and Disability, 14(3):101-105.

Dollar, A. M. and Howe, R. D. (2010). The highly adaptive sdm hand: Design and performance evaluation. The international journal of robotics research, 29(5):585-597.

Dollar, A. M. and Howe, R. D. (2011). Joint coupling design of underactuated hands for unstructured environments. The International Journal of Robotics Research, 30(9):1157-1169.

Eppner, C. and Brock, O. (2013). Grasping unknown objects by exploiting shape adaptability and environmental constraints. In Intelligent Robots and Systems (IROS), 2013 IEEE/RSJ International Conference on, pages 4000-4006.

Falco, J., Van Wyk, K., Liu, S., and Carpin, S. (2015). Grasping the performance: Facilitating replicable performance measures via benchmarking and standardized methodologies. Robotics \& Automation Magazine, IEEE, 22(4):125-136.

Farina, D. and Merletti, R. (2000). Comparison of algorithms for estimation of emg variables during voluntary isometric contractions. Journal of Electromyography and Kinesiology, 10(5):337-349.

Felzer, T. and Freisleben, B. (2002). Ilawcos: the hands-free wheelchair control system. In Proceedings of the fifth international ACM conference on Assistive lechnologies, pages 127-134. ACM.

Gafford, J., Ding, Y., Harris, A., McKenna, T., Polygerinos, P., Holland, D., Moser, A., and Walsh, C. (2014). Shape deposition manufacturing of a soft, atraumatic, deployable surgical grasper. Journal of Medical Devices, 8(3):030927.

Gillen, G. (2015). Stroke rehabilitation: a function-based approach. Elsevier Ilealth Sciences.

Go, A. S., Mozaffarian, D., Roger, V. L., Benjamin, E. J., Berry, J. D., Blaha, M. J., Dai, S., Ford, E. S., Fox, C. S., Franco, S., ct al. (2014). Heart discase and stroke statistics-2014 update: a report from the american heart association. Circulation, 129(3): $\mathrm{c} 28$.

Graf, C. (2008). The lawton instrumental activitics of daily living scalc. AJN The American Journal of Nursing, 108(4):52-62.

IIeo, P., Gu, G. M., Lec, S.-j., Rhec, K., and Kim, J. (2012). Current hand exoskeleton technologies for rehabilitation and assistive enginecring. International Journal of Precision Engineering and Manufacturing, 13(5):807-824. 
Holland, D. P., Park, E. J., Polygerinos, P., Bennett, G. J., and Walsh, C. J. (2014). The soft robotics toolkit: shared resources for research and design. Soft Robotics, 1(3):224-230.

Hussain, I., Meli, L., Pacchierotti, C., Salvietti, G., and Prattichizzo, D. (2015a). Vibrotactile haptic fedback for intuitive control of robotic extra fingers. In Proc. IEEE World Haptics Conference (WHC), Chicago, IL.

Hussain, I., Salvietti, G., Malverzi, M., and Prattichizro, D. (2017a). On the role of stiffness design for fingertip trajectories of underactuated modular soft hands. In Proc. IEEE Int. Conf. on Robotics and Automation, Singapore.

IIussain, I., Salvietti, G., Meli, L., Pacchierotti, C., and Prattichizzo, D. (2015b). Using the robotic sixth finger and vibrotactile feedback for grasp compensation in chronic stroke patients. In Proc. IEEE/RAS-EMBS International Conference on Rehabilitation Robotics (ICORR).

Iussain, I., Salvietti, G., Spagnoletti, G., Malvezzi, M., Cioncoloni, D., Rossi, S., and Prattichizzo, D. (2017b). A soft supernumerary robotic finger and mobile arm support for grasping compensation and hemiparetic upper limb rehabilitation. Robotics and Autonomous Systems, 93:1 - 12.

Iussain, I., Salvictti, G., Spagnoletti, G., and Prattichizzo, D. (2016). The soft-sixthfinger: a wearable emg controlled robotic extra-finger for grasp compensation in chronic stroke patients. IEEE Robotics and Automation Letters, 1(2):1000-1006.

Kiguchi, K., Tanaka, T., and Fukuda, 'T. (2004). Ncuro-fuzzy control of a robotic exoskeleton with $\mathrm{emg}$ signals. Fuzzy Systems, IEEE Transactions on, 12(4):481-490.

Konrad, P. (2005). The abc of emg. A practical introduction to kinesiological electromyography, 1.

Laschi, C. and Cianchetti, M. (2014). Soft robotics: new perspectives for robot bodyware and control. Frontiers in bioengineering and biotechnology, 2(3).

Lum, P. S., Godfrcy, S. B., Brokaw, E. B., Holley, R. J., and Nichols, D. (2012). Robotic approaches for rehabilitation of hand function after stroke. American Journal of Physical Medicine \& Rehabilitation, 91(11):S242-S254.

Lund, A. M. (2001). Measuring usability with the use questionnaire. Usability interface, 8(2):3-6.

Ma, R. R., Belter, J. T., and Dollar, A. M. (2015). Ilybrid deposition manufacturing: design strategies for multimaterial mechanisms via threc-dimensional printing and material deposition. Journal of Mechanisms and Robotics, 7(2):(021002.

Meng, Q. and Lec, M. H. (2006). Design issues for assistive robotics for the elderly. Advanced engineering informatics, 20(2):171-186.

Merletti, R., Botter, A., Troiano, A., Merlo, E., and Minctto, M. A. (2009). Technology and instrumentation for detection and conditioning of the surface electromyographic signal: state of the art. Clinical Biomechanics, 24(2):122-134.

Merlo, A. and Campanini, I. (2010). Technical aspects of surface electromyography for clinicians. The Open Rehabilitation Journal, 3(1).

Michaelsen, S. M., Jacobs, S., Roby-Brami, A., and Levin, M. F. (2004). Compensation for distal impairments of grasping in adults with hemiparesis. Experimental Brain Research, 157(2):162-173.

Migueleı, J., Miguele», M., and Alley, R. (2004). Amputations about the shoulder: prosthetic management. Altas of Amputations and Limb DeficienciesSurgical, Prosthetic, and Rehabilitation Principles. Rosemont, IL: American Academy or Orthopaedic Surgeons, pages 263-273.

Nakayama, II., Jorgensen, II. S., Raaschou, II. O., and Olsen, T. S. (1994). Compensation in recovery of upper extremity function after stroke: the copenhagen stroke study. Archives of physical medicine and rehabilitation, 75(8):852-857.

Nowak, D. A. (2008). The impact of stroke on the performance of grasping: usefulness of kinetic and kinematic motion analysis. Neuroscience \& Biobehavioral Reviews, 32(8):1439-1450.

Odhner, L. U., Jentoft, L. P., Claffee, M. R., Corson, N., Tenzer, Y. Ma, R. R., Buehler, M., Kohout, R., Howe, R. D., and Dollar, A. M. (2014). A compliant, underactuated hand for robust manipulation. The International Journal of Robotics Research, $33(5): 736-752$.

Ort, T., Wu, F., IIensel, N. C., and Asada, Il. H. (2015). Supernumerary robotic fingers as a therapeutic device for hemiparetic patients. In ASME 2015 Dynamic Systems and Control Conference, pages V002T27A010-V002T27A010. American Society of Mechanical Enginecrs.

Oskoci, M. A. and Hu, H. (2007). Myoelectric control systems - a survey. Biomedical Signal Processing and Control, 2(4):275294.

Pons, J., Rocon, E., Ceres, R., Reynaerts, D., Saro, B., Levin, S., and Van Moorleghem, W. (2004). The manus-hand dextrous robotics upper limb prosthesis: mechanical and manipulation aspects. Autonomous Robots, 16(2):143-163.

Pons, J. L. (2010). Rehabilitation cxoskclctal robotics. Engineering in Medicine and Biology Magazine, IEEE, 29(3):57-63.

Pons, J. L. et al. (2008). Wearable robots: biomechatronic exoskeletons, volume 338. Wiley Online Library.

Prattichizzo, D., Malvezzi, M., Ilussain, I., and Salvietti, G. (2014a). The sixth-finger: a modular extra-finger to enhance human hand capabilitics. In Proc. IEEE Int. Symp. in Robot and IIuman Interactive Communication, Edinburgh, United Kingdom. 
Prattichizzo, D., Salvietti, G., Chinello, F., and Malvezzi, M. (2014b). An object-based mapping algorithm to control wearable robotic extra-fingers. In Proc. IEEE/AS'ME Int. Conf. on Advanced Intelligent Mechatronics, Besançon, France.

Raghavan, P., Krakauer, J. W., and Gordon, A. M. (2006). Impaired anticipatory control of fingertip forces in patients with a pure motor or sensorimotor lacunar syndrome. Brain, 129(6):14151425.

Salvietti, G., IIussain, I., Cioncoloni, D., Taddei, S., Rossi, S., and Prattichizzo, D. (2016). Compensating hand function in chronic stroke patients through the robotic sixth finger. Transaction on Neural System and Rehabilitation Enginecring.

Saponas, T. S., Tan, D. S., Morris, D., and Balakrishnan, R. (2008). Demonstrating the feasibility of using forearm electromyography for muscle-computer interfaces. In Proceedings of the SIGCIII Conference on IIuman Factors in Computing Systems, pages 515-524. ACM.

Schwarz, R. J. (1955). The anatomy and mechanics of the human hand. Artificial Limbs, 22.

Stanger, C. A., Anglin, C., Harwin, W. S., and Romilly, D. P. (1994). Devices for assisting manipulation: a summary of user task priorities. Rehabilitation Engineering, IEEE Transactions on, 2(4):256-265.

T Walley Williams IU, M., Altobelli, D. L., et al. (2011). Prosthetic sockets stabilized by alternating areas of tissue compression and releasc. Journal of rehabilitation research and development, 48(6):679.

Timoshenko, S. and Gere, J. (1972). Mechanics of materials. Van Nostrand Reinhold Co.

Van der Loos, II. M. and Reinkensmeyer, D. J. (2008). Rehabilitation and health carc robotics. In Springer Ilandbook of Robotics, pages 1223-1251. Springer.

Vanderborght, B., Albu-Schäffer, A., Bicchi, A., Burdet, E., Caldwell, D. G., Carloni, R., Catalano, M., Eiberger, O., Fricdl, W., Ganesh, G., et al. (2013). Variable impedance actuators: A revicw. Robotics and autonomous systems, 61(12):1601-1614.

Webb, J., Xiao, Z. G., Aschenbrenner, K. P., IIerrnstadt, G., and Menon, C. (2012). Towards a portable assistive arm cxoskcleton for stroke patient rehabilitation controlled through a brain computer interfacc. In Biomedical Robotics and Biomechatronics (BioRob), 2012 4th IEEE RAS \& EMBS International Conference on, pages 1299-1304. IEEE.

Wu, F. and $\Lambda$ sada, H. (2014). Bio-artificial synergics for grasp posture control of supernumerary robotic fingers. In Proceedings of Robotics: Science and Systems, Bcrkelcy, USA.

Wu, F. Y. and $\Lambda$ sada, H. H. (2016). Implicit and intuitive grasp posture control for wearable robotic fingers: $A$ data-driven method using partial least squares. IEEE Transactions on
Robotics, 32(1):176-186.

Zecca, M., Micera, S., Carrozza, M., and Dario, P. (2002). Control of multifunctional prosthetic hands by processing the electromyographic signal. Critical Reviews in Biomedical Engineering, 30(4-6).

ArbotiX (2012). Arbotix-m robocontroller,open source. On-line: htlp://www.trossenrobotics.com/p/arbotix-robotcontroller.aspx.

Robotis (2012). Dynamixel mx-281 robot actuator. Online: http://www.trossenrobolics.com/dynamixel-mx-28-robotactuator.aspx. 